\title{
Carbon and oxygen isotope composition of Cambro-Silurian limestone and anthraconite from Bornholm: Evidence for deep burial diagenesis.
}

\author{
BJøRN BUCHARDT and ARNE THORSHøJ NIELSEN
}

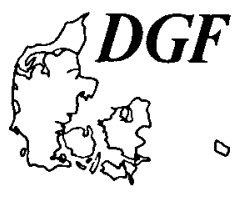

Buchardt, B. and Nielsen, A. T.: Carbon and oxygen isotope composition of Cambro-Silurian limestone and anthraconite from Bornholm: Evidence for deep burial diagenesis. Bull. geol. Soc. Denmark, vol. 33 pp. 415-435, Copenhagen, February, 28th, 1985.

\begin{abstract}
The Cambro-Silurian clastic deposits of the Island of Bornholm in the Baltic Sea include carbonate rocks developed as primary limestone, early diagenetic concretions (mainly anthraconite) and late diagenetic vein and cavity fillings. Oxygen isotope compositions of primary limestone and concretions are lithologically and stratigraphically homogenized to values significantly different from those of diagenetically unaffected marine carbonate. Instead, the composition is explained as a result of burial diagenesis at temperatures in excess of $90^{\circ} \mathrm{C}$. On the other hand, the same rocks have retained their original carbon isotope compositions. The values reflect differences between carbonate precipitated in equilibrium with marine bicarbonate and carbonate formed in the sulphate reduction zone of the bottom sediments. The vein- and cavity fillings have isotopic compositions which are deviating markedly from the other material. Depending on choice of geothermal gradient and primary, depositional parameters the observed thermal impact can be explained from burial of the Lower Palaeozoic sequence to depths greater than $2 \mathbf{~ k m}$. It is suggested that this subsidence took place in Late Silurian to Earliest Devonian time in relation to the major Caledonian phase.
\end{abstract}

Bjørn Buchardt and Arne Thorshøj Nielsen, Iustitute of Historical Geology and Palaeontology, Øster Voldgade 10, DK-1350 Copenhagen K, Denmark. November 20th, 1984.

Contribution to EFP-83 no. 1.

\section{Introduction}

The Island of Bornholm in the Baltic Sea includes a Lower Palaeozoic sedimentary sequence of approximately $500 \mathrm{~m}$ of which Lower Cambrian coarse and medium-grained clastic deposits constitute more than half. The dominant lithology above the Lower Cambrian is shale and mudstone including minor intercalation of limestone. A few kilometers south of Bornholm, in the Rønne Graben, seismic recordings reveal the presence of more than $2.2 \mathrm{sec}$ (TWT) of presumed Palaeozoic deposits (Vejbæk, in press), which in thickness corresponds to 4 to $5 \mathrm{~km}$.

The post-Lower Palaeozoic rocks of Bornholm comprise Mesozoic deposits of predominantly clastic character often covering weathered Palaeozoic and Precambrian rocks, as well as thin Quaternary deposits. The total thickness of the Post-Palaeozoic sequence is less than $1.5 \mathrm{~km}$ in the onshore sections, while the corresponding thickness in the Rønne Graben amounts to 2 to 3 $\mathrm{km}$.
In the present study, a survey has been carried out of the stable isotope composition of the Cambro-Silurian carbonate rocks of Bornholm. The aim of the study has been to investigate the diagenetic history of the sequence. Carbonates occur in the interval as cement, concretions, true limestone units up to approximately $5 \mathrm{~m}$ in thickness and vein and cavity fillings. It is shown that these groups exhibit clear differences with regard to the ${ }^{13} \mathrm{C} /{ }^{12} \mathrm{C}$-ratios, but no differences in ${ }^{18} \mathrm{O} /{ }^{16} \mathrm{O}$ ratios except for the late diagenetic vein fillings. Moreover, the carbonates are depleted in ${ }^{18} \mathrm{O}$ as compared to "normal" marine carbonates. These facts cannot be explained as a result of primary depositional and early diagenetic conditions alone, and a deep burial history is favoured as a possible explanation of the present pattern of isotopic values. It is suggested that the thick Palaeozoic sequence-now seen south of Bornholm originally covered the whole area, but later suffered extensive erosion, probably in Late Palaeozoic time. This hypothesis also explains the postmature state with regard to oil-generation of the 
SCHEME 1

SCHEME 2

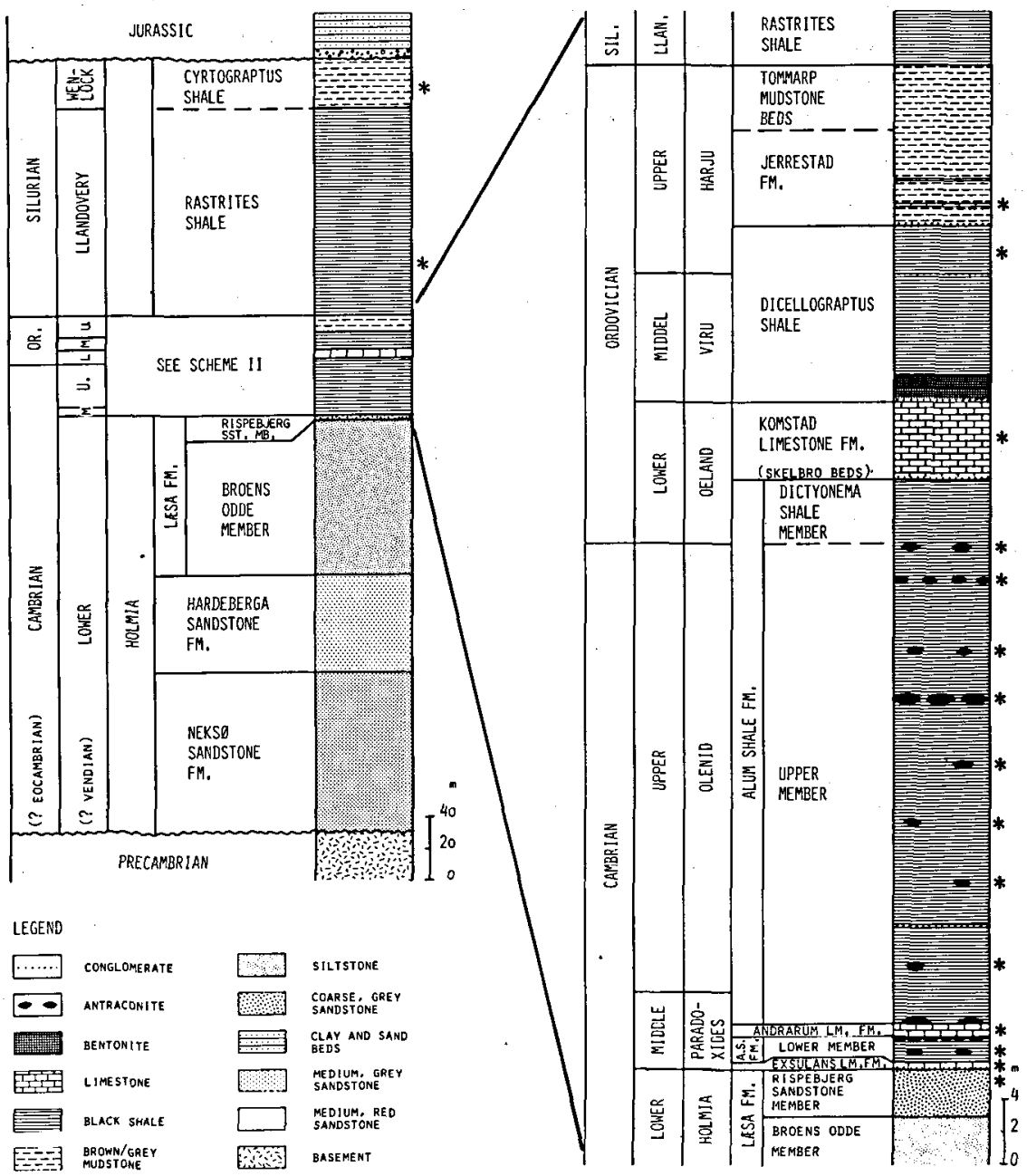

Figure 1. Lithostratigraphical schemes of the investigated Cambro-Silurian deposits of Bornholm. Chronostratigraphy in accordance with V. Poulsen (1966). Scheme II at the right gives an expanded version of the Cambro-Ordovician part of scheme I. Note the different vertical scales for the two colums. ${ }^{*}$ denotes sampled horizons.

Middle and Upper Cambrian black shales (Thomsen et al., 1983).

\section{Geological setting}

The Cambro-Silurian stratigraphy of Bornholm was revised and updated by V. Poulsen (1966): A recent summary is found in Surlyk (1980). In the following a brief outline is given of the present lithostratigraphical concept of the Lower Palaeozoic deposits of Bornholm. The units are referred to serie-level on the basis of the information given by V. Poulsen (1966), see fig. 1 . The investigated units are in ascending order:

\section{Vendian?/Early Cambrian}

1. Nexø Sandstone Fm.: $100 \mathrm{~m}$ of subarcosic, fluviatile red sandstone devoid of carbonates. The unit is not represented in the present isotopic study.

2. Hardeberga Sandstone Fm. (senior synonym for Balka Sandstone, V. Poulsen (1978a)): $60 \mathrm{~m}$ of marine, partly glauconitic sandstone devoid of carbonates. 
3. Læså fm. (Surlyk 1980): $100 \mathrm{~m}$ of marine, glauconitic siltstones and fine-grained sandstones (Broens Odde member) overlain by 2 to $3 \mathrm{~m}$ of coarse-grained sandstone (Rispebjerg member). Several lithologies of the Læså fm. (both members) have carbonate cement. A few analyses of the cement are given here.

\section{Middle Cambrian}

4. Exsulans Limestone Fm.: $0.2 \mathrm{~m}$ of dense grey, in parts strongly glauconitic limestone. A weathered counterpart, the socalled "Kalby Marl«, is found at the small stream Læså (Berg-Madsen 1981).

5. Alum Shale Fm. (sensu Gee 1972), lower member (here replacing the old term "Lower Alum Shale ): 0.8 to $1.5 \mathrm{~m}$ of black, organic-rich shale with a few lenses of black, bituminous concretionary limestone (anthraconite).

6. Andrarum Limestone Fm.: 0.5 to $0.8 \mathrm{~m}$ of grey, dense biomicritic to biosparitic limestone with several discontinuity surfaces. Minor parts of the unit are dark-grey and resemble anthraconite.

\section{Late Cambrian}

7. Alum Shale Fm., upper member (here replacing the old term »Upper Alum Shale«): Approx. $30 \mathrm{~m}$ black, organic-rich shale, of which the lowermost two metres belong to the Middle Cambrian. The unit contains several lenses and layers of anthraconite at different levels.

\section{Early Ordovician}

8. Alum Shale Fm., Dictyonema Shale member (not a strict lithostratigraphical unit, the lower boundary is defined on palaeontological evidence): $4 \mathrm{~m}$ of black, organic-rich shale resembling the lower units of the Alum Shale Fm. The unit lacks anthraconite.

9. Komstad Limestone Fm. (replacing the old term »Orthoceras Limestone«): $5 \mathrm{~m}$ of grey, highly lithified micritic limestone with a clay content between 5 and $20 \%$. A few clay-seems are found in the middle part. The unit contains several discontinuity surfaces. The limestone was formerly subdivided into a lower Skelbro Fm. and an upper Komstad Fm. (V. Poulsen 1965).
This separation cannot be upheld (Nielsen in prep.), and the Skelbro Fm. sensu V. Poulsen (1965) is here included in the Komstad Limestone Fm. under the name Skelbro beds.

Middle and Late Ordovician

10. »Dicellograptus Shale«: $11 \mathrm{~m}$ of dark, partly bioturbated shale with a few bentonite horizons. Carbonate layers and lenses are generally absent, but a single anthraconitic lens has been included in the present study.

11. Jerrestad Fm. (formerly »Tretaspis Shale «): 4 to $8 \mathrm{~m}$ of grey to light brown, not distinctly fissile mudstone. Two thin calcite-cemented mudstone layers are found in the lower part of the unit.

12. Tommarp Mudstone Fm. (formerly Dalmanitina beds): 2 to $6 \mathrm{~m}$ of light-grey mudstone rich in fossils. The unit is known only from drill cuttings (Baungegård well section, V. Poulsen 1978b) and is not included in the present study.

\section{Early Silurian}

13. »Rastrites Shale«: $135 \mathrm{~m}$ of dark-grey mudstone with subordinate black, organic-rich horizons. Both true limestone layers and concretionary bodies are described from the unit, (Bjerreskov 1975).

\section{Middle Silurian}

14. »Cyrtograptus Shale«: $25 \mathrm{~m}$ of light-grey shaly mudstone with occasional lenses of concretionary limestone.

In general, the sequence can be interpreted as representing at least two major tectonically induced deepenings and transgressions followed by coastline progradation (Surlyk 1980). The first cycle includes the Eocambrian/Lower Cambrian coarse-clastic deposits, the second cycle comprises the dominantly clayey deposits of Middle Cambrian to Middle Silurian age. The several hiati within the latter cycle may represent minor regressiv episodes followed by emergence and erosion, but at least part of them probably are caused by submarine nondeposition and erosion.

The depositional environment is believed to be fully marine with only minimal influence from fresh water. The generally euxinic character of 
the Middle Cambrian to Lower Ordovician noncalcareous deposits can be interpreted as a result of restricted fjord-like conditions, but the regionwide extent of the black-shale units contradicts this. Probably, the depositional environment through most of the second cycle was that of an outer shelf, where regional patterns of ocean current systems combined with high organic productivity gave rise to the extensive anoxic deposits.

\section{Material}

In the present investigation, 71 samples of Cambro-Silurian carbonates have been analysed for their carbon and oxygen isotope compositions. Most samples have been collected from exposures along the small streams Læså, Øleå and Risebæk and at the abandoned limestone quarry at Skelbro (see fig. 2). In addition, material has been included from a shallow core (Vasegård I) drilled by the Geological Survey of Greenland at a locality close to Læså. Where possible, in situ material has been preferred, but biostratigraphically well-dated loose material from the stream bottoms has been included as well. The stratigraphical position of the material has been determined in accordance with Grönwall (1902), C. Poulsen (1923), V. Poulsen (1966), and Bjerreskov (1975).

The investigated material can be grouped into four categories according to lithology: primary limestone, calcite-cemented mudstone, concretionary limestone (anthraconites) and late diagenetic carbonates.

The category 'primary limestone' includes the Middle Cambrian Exsulans and Andrarum Limestone units and the Lower Ordovician Komstad Formation. They are light- to dark-grey, clayrich, highly lithified biomicrites and biosparites developed as well-bedded units. The dominant mineral is calcite. Moreover, pyrite, glauconite and at some horizons phosphorite nodules are found. The limestone units contain several synsedimentary dissolution horizons and probable hardgrounds, traits that support the concept of a primary, depositional origin for this material.

The category 'calcite-cemented mudstone' comprises material from the Upper Ordovician Jerrestad Fm. and from the Lower and Middle Silurian units. The Jerrestad Fm. material in-

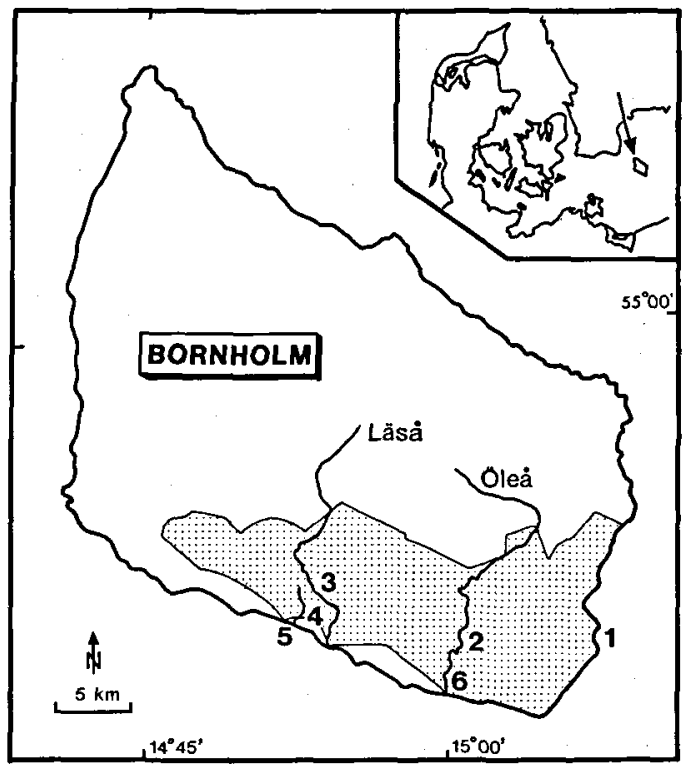

Figure 2. Geographical location of the island of Bornholm in the Baltic Sea. Dotted area indicates outcrop of Lower Palaeozoic deposits under thin Quaternary cover. The following localities have been sampled. 1: Broens Odde, Lower Cambrium; 2: Borregård, Øleå, Lower to Upper Cambrium; 3: Kalby and Vasegård, Laså, Middle to Upper Cambrium; 4: Skelbro Quarry Lower Ordovicium; 5: Risebæk, Upper Ordovicium; and 6: Køllegård to Sommerodde, Øleả, Lower to Middle Silur.

cludes samples from two close-lying horizons, less than $10 \mathrm{~cm}$ thick, of microsparitic to micritic, grey-brown mudstone. It is structureless except for several uncompacted trace fossils of the Chondrites-type. The Silurian material originates from similar horizons, of which the Lower Silurian ones are the thickest and most numerous. The genetic history of the material is unclarified, but probably represents episodes of early diagenetic carbonate cementation of the muddy sediment.

The category 'concretionary limestone' includes black, sparitic to microsparitic anthraconite developed either as lenticular bodies up to $1.5 \mathrm{~m}$ in horizontal diameter or as beds up to 0.3 $\mathrm{m}$ in thickness. The anthraconitic limestone is composed of calcite, clay, pyrite and organic material (up to $8 \%$; Buchardt \& Cederberg in prep.). The internal structures of the concretionary bodies such as uncompacted fossils, primary mud-bottom lamination that is commonly horizontal in the central part and curved in the marginal parts of the concretionary bodies, and cone- 
in-cone structures marginally, as well as compaction structures in the surrounding shales, suggest an early diagenetic, pre- to syncompactional origin for this material (cf. discussion in Raiswell 1971). Stratigraphically the anthraconite is confined to the black shale deposits or to the boundary horizons between shale and limestone units. It occurs abundantly in the Middle and Upper Cambrian Alum Shale Formation, but is found in the Middle to Upper Ordovician Dicellograptus Shale as well.

The category 'late diagenetic carbonate' includes sparitic vein and cavity fullings found both in the primary limestone units and in the anthraconitic bodies, and calcitic cement found in subordinate horizons of the Lower Cambrian siltand sandstones of the Lxas formation. Moreover, carbonate crusts of modern age formed on the exposed surfaces of the Komstad Limestone in the Skelbro Quarry have been analysed.

\section{Analytical methods}

Calcium carbonate for isotopic determinations has been sampled by aid of a dental drill from freshly cut surfaces of the investigated rocks. In case of visual inhomogeneities, samples have been drilled from the most representative areas. A special problem is imposed by the possibility of isotopic inhomogenenties of the concretions, see e.g. Coleman \& Raiswell (1981). In most cases, samples have been selected from the central, fine-crystalline part of the anthraconitic bodies. However, four bodies have been sampled in detail in order to investigate any zoning in isotopic composition.

Most exposures are affected by weathering. For carbonates, this effect normally is limited to the outermost few $\mathrm{cm}$ of the rocks owing to the highly impermeable nature of the fine-grained and often organic-rich material. The effect of weathering on the isotopic composition has been evaluated from analyses of strongly weathered and corresponding well-preserved samples.

The calcium carbonate content of the material was determined volumetrically (accuracy better than $\pm 2 \%$ ), while the mineralogical composition was evaluated by X-ray diffraction. For isotopic analyses the material was treated in accordance with the standard methods described by McCrea
(1950). After grinding to a grain size between 150 and $75 \mu \mathrm{m} 30 \mathrm{mg}$ of carbonate powder was dissolved in $10 \%$ hydrophosphoric acid in vacuo at $25.0^{\circ} \mathrm{C}$. The ${ }^{13} \mathrm{C} /{ }^{12} \mathrm{C}$ - and ${ }^{18} \mathrm{O} /{ }^{16} \mathrm{O}$-ratios of the evolved carbon dioxide gas were measured in a Finnigan MAT 250 triple collector mass spectrometer. In the following, both carbon and oxygen isotope ratios are expressed as per mille deviation from the PDB-standard using the $\delta$-function (Epstein et al. 1951). Reproducibility is better than $\pm 0.03 \%$ on the $\delta$-scale for both ratios.

\section{Results}

\section{Mineralogy and calcium carbonate content}

The carbonate phase of the investigated rocks is low-Mg calcite. Calcium carbonate concentration in primary limestone samples vary between $\mathbf{5 0}$ and $86 \%$ with additional clay, glauconite, phosphorite and pyrite. Anthraconites have calcium carbonate content from 60 to $90 \%$, most samples being in the range above $80 \%$. This supports the interpretation of the anthraconites as being formed in an early diagenetic environment, where porosity available for carbonate cementation can be up to $90 \%$ (Raiswell, 1971). The calcite-cemented mudstones have varying carbonate content generally lower than the primary limestone and anthraconite.

\section{Isotopic composition}

The analytical results are presented in table 1 and 2 and figs $3,4,5$. In order to evaluate the reliability of the isotopic data the influence from weathering and the range of intra-unit variablility has been determined.

\section{Effect of weathering}

The effect of isotopic exchange related to weathering can be evaluated from table 3 . The most straight-forward approach is to compare the relatively well-preserved Exsulans Limestone with the Kalby Clay. As seen in fig. 3, the Kalby Clay is enriched in ${ }^{18} \mathrm{O}$ as compared to the Exsulans Limestone. This is to be expected from exchange processes acting between the strongly ${ }^{18} \mathrm{O}$-de- 
Table 1. Carbon and oxygen isotope composition of the investigated materiale.

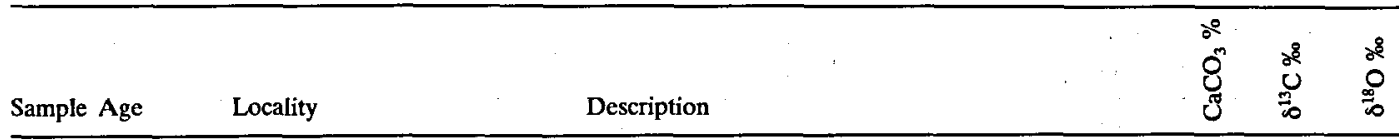

\section{Exsulans limestone formation}

E-1 M.Camb. Borregård, Øleå

E-2 M.Camb. Borregård, Øleå

E-3 M.Camb. Borregård, Øleå

E-4 M.Camb. Borregård, Øleå

E-5 M.Camb. Kalby, Laså

E-6 M.Camb. Kalby, Laså

E-7 M.Camb. Kalby, Laså

E-8 M.Camb. Kalby, Laså

Andrarum limestone formation

AL-1 M.Camb. Kalby, Kæså

AL-2 M.Camb. Borregård, Øleå

AL-3 M.Camb. Borregård, Øleå

AL-4 M.Camb. Borregård, Øleå

Komstad limestone formation

K-1 L.Ordv. Limensgade, Læså

K-2 L.Ordv. Limensgade, Læså

K-3 L.Ordv. Skelbro Quarry

K-4 L.Ordv. Skelbro Quarry

K-5 L.Ordv. Skelbro Quarry

K-6 L.Ordv. Skelbro Quarry

K-7 L.Ordv. Skelbro Quarry

K-8 L.Ordv. Skelbro Quarry

K-9 L.Ordv. Skelbro Quarry

K-10 L.Ordv. Vasegård I, core

K-11 L.Ordv. Vasegård I, core

K-12 L.Ordv. Vasegård I, core

K-13 L.Ordv. Vasegård I, core

K-14 L.Ordv. Vasegård I, core

K-15 L.Ordv. Vasegård I, core

Tretaspis limestone

T-1 U.Ordv. Vasegård, Laså
Micritic to microsparitic grey limestone with

shell debris ........................... $85 \quad-2.11 \quad-8.13$

Micritic, grey limestone ..................... $47 \quad-1.66 \quad-8.59$

Sparitic to microsparitic, grey limestone........... $86 \quad \begin{array}{llll}-0.04 & -9.35\end{array}$

Micritic, grey limestone with shell debris.......... $83 \quad-0.68 \quad-11.11$

Kalby Clay, total sample $\ldots \ldots \ldots \ldots \ldots \ldots \ldots \ldots \ldots \ldots, 64 \quad-1.22 \quad-8.99$

Kalby Clay, fraction $>75$ micron, data from

Berg-Madsen (1981) ..................... - $-2.95 \quad-9.28$

Kalby Clay, fraction $>75$ micron $\ldots \ldots \ldots \ldots \ldots \ldots \ldots . .67 \quad-2.74 \quad-8.97$

Kalby Clay, echinoderm fragments, data from

Berg-Madsen (1981) ...................... - $-2.46 \quad-7.06$

Microsparitic to micritic, grey limestone........... $79 \quad-2.84 \quad-11.84$

Microsparitic to micritic, grey limestone........... $86 \quad-1.83 \quad-10.73$

Microsparitic, dark-grey limestone.............. $76 \quad-1.72 \quad-11.28$

Microsparitic, dark-grey limestone with abundant

shell fragments......................... $77 \quad-1.86 \quad-11.67$

Calcitic cement in conglomerate, grey, with glauconite

(Skelbro beds) .......................... $68 \quad-4.22 \quad-11.80$

Micritic, grey limestone, $10 \mathrm{~cm}$ above base of

conglomerate (Skelbro beds) $\ldots \ldots \ldots \ldots \ldots \ldots \ldots \ldots \ldots 73 \quad-3.22 \quad-11.09$

Micritic, grey limestone, $15 \mathrm{~cm}$ above base of

conglomerate (Skelbro beds)................. $77 \quad-3.06 \quad-11.14$

Microsparitic, grey limestone, $31 \mathrm{~cm}$ above top of

conglomerate $\ldots \ldots \ldots \ldots \ldots \ldots \ldots \ldots \ldots \ldots \ldots \ldots, 75 \quad-1.17 \quad-10.52$

Micritic to microsparitic, grey limestone, $96 \mathrm{~cm}$ above

top of conglomerate $\ldots \ldots \ldots \ldots \ldots \ldots \ldots \ldots \ldots \ldots, 67-1.03 \quad-11.32$

Micritic, light grey limestone, $170 \mathrm{~cm}$ above top of conglomerate $\ldots \ldots \ldots \ldots \ldots \ldots \ldots \ldots \ldots \ldots \ldots \ldots .64 \quad-1.08 \quad-11.34$

Micritic, light grey limestone, $247 \mathrm{~cm}$ above top of conglomerate ............................ $76 \quad-0.64 \quad-11.87$

Micritic, dark grey limestone, $296 \mathrm{~cm}$ above top of

conglomerate $\ldots \ldots \ldots \ldots \ldots \ldots \ldots \ldots \ldots \ldots \ldots \ldots \ldots \ldots, 72 \quad-0.29 \quad-11.31$

Micritic, grey limestone, $343 \mathrm{~cm}$ above top of

conglomerate $\ldots \ldots \ldots \ldots \ldots \ldots \ldots \ldots \ldots \ldots \ldots \ldots \ldots .71 \quad-1.20 \quad-11.44$

Microsparitic, grey limestone, $30 \mathrm{~cm}$ above top of

conglomerate $. \ldots \ldots \ldots \ldots \ldots \ldots \ldots \ldots \ldots \ldots \ldots .50 \quad-1.26 \quad-11.41$

Micritic, dark grey limestone, $94 \mathrm{~cm}$ above top of conglomerate $\ldots \ldots \ldots \ldots \ldots \ldots \ldots \ldots \ldots \ldots \ldots \ldots \ldots .72-0.66 \quad-11.72$

Micritic, light-grey limestone, $170 \mathrm{~cm}$ above top of conglomerate $. . \ldots \ldots \ldots \ldots \ldots \ldots \ldots \ldots \ldots, \ldots \ldots, 29,-0.45-11.05$

Micritic, grey limestone, $226 \mathrm{~cm}$ above top of conglomerate $\ldots \ldots \ldots \ldots \ldots \ldots \ldots \ldots \ldots \ldots \ldots \ldots .73 \quad-0.88 \quad-12.12$

Micritic, dark grey limestone, $291 \mathrm{~cm}$ above top of conglomerate $\ldots \ldots \ldots \ldots \ldots \ldots \ldots \ldots \ldots \ldots \ldots \ldots \ldots .71 \quad-0.62 \quad-12.04$

Micritic, grey limestone, $374 \mathrm{~cm}$ above top of

conglomerate ........................... $81 \quad-0.49 \quad-11.65$

Micritic, silicified, homogeneous limestone,

Jerrestad Fm........................... $62 \quad-5.30 \quad-11.07$ 


\begin{tabular}{|c|c|c|c|c|c|c|}
\hline Sample & Age & Locality & Description & $\delta_{0}^{\circ}$ & $\bigcup_{0}^{8}$ & $\begin{array}{l}x^{8} \\
0 \\
\infty \\
\infty\end{array}$ \\
\hline$T-2$ & U.Ordv. & Vasegård, Læså & $\begin{array}{l}\text { Micritic, silicified, homogeneous limestone, } \\
\text { Jerrestad Fm...................... }\end{array}$ & 58 & -5.07 & -10.87 \\
\hline $\mathrm{T}-3$ & U.Ordv. & Vasegård, Læså & $\begin{array}{l}\text { Micritic, silicified, homogeneous limestone, } \\
\text { bioturbated, Jerrestad Fm. . . . . . . . . . . . . . . }\end{array}$ & 54 & -5.25 & -11.27 \\
\hline \multicolumn{7}{|c|}{ Silurian concretionary limestone } \\
\hline L-1 & Lland. & Køllergård, Øleå & $\begin{array}{l}\text { Microsparitic, dark grey, laminated, concretionary } \\
\text { limestone bed } \ldots \ldots \ldots \ldots \ldots \ldots \ldots \ldots \ldots \ldots \ldots \ldots \ldots \ldots \ldots\end{array}$ & 83 & +1.00 & -10.58 \\
\hline L-2 & Lland. & Køllergård, Øleå & $\begin{array}{l}\text { Microsparitic to micritic, laminated concretionary } \\
\text { limestone bed. } \ldots \ldots \ldots \ldots \ldots \ldots \ldots \ldots \ldots \ldots \ldots \ldots\end{array}$ & 83 & +0.70 & -10.25 \\
\hline W-1 & U.Wenlock & Sommerodde, Øleå & Micritic, homogenous, concretionary limestone ...... & 39 & -1.85 & -9.10 \\
\hline W-2 & U.Wenlock & Sommerodde, Øleå & Calcareous mudstone, homogeneous............... & 19 & -1.89 & -8.16 \\
\hline \multicolumn{7}{|c|}{ Anthraconites } \\
\hline $\begin{array}{l}A-1 \\
A-2\end{array}$ & $\begin{array}{l}\text { M.Camb. } \\
\text { M.Camb. }\end{array}$ & $\begin{array}{l}\text { Kalby, Lxså } \\
\text { Borregård, Øleå }\end{array}$ & $\begin{array}{l}\text { Sparitic anthraconite, weathered, above Kalby Clay ... } \\
\text { Sparitic, fine-grained, homogeneous anthraconite, } \\
\text { Alum Shale Fm., lower member } \ldots \ldots \ldots \ldots \ldots \ldots \ldots \ldots\end{array}$ & 88 & $\begin{array}{l}-5.50 \\
-5.43\end{array}$ & $\begin{array}{r}-9.61 \\
-12.14\end{array}$ \\
\hline A-3 & M.Camb. & Borregård, Øleå & $\begin{array}{l}\text { Sparitic anthraconite, Alum Shale Fm., lower member, } \\
\text { average }{ }^{*} \ldots \ldots \ldots \ldots \ldots \ldots \ldots \ldots \ldots \ldots \ldots \ldots \ldots \ldots\end{array}$ & 87 & -4.98 & -10.66 \\
\hline A-4 & M.Camb. & Borregård, Øleå & $\begin{array}{l}\text { Sparitic, fine- to medium-grained anthraconite, } \\
\text { Alum Shale Fm., lower member, average* } \ldots \ldots \ldots \ldots\end{array}$ & 85 & -3.42 & -8.97 \\
\hline A.5 & M.Camb. & Laså & $\begin{array}{l}\text { Sparitic, fine-grained, laminated anthraconite, } \\
\text { immediately below Andrarum } \mathrm{Fm} . \ldots \ldots \ldots \ldots \ldots \ldots\end{array}$ & 81 & -4.27 & -11.41 \\
\hline A-6 & M.Camb. & Kalby, Lxså & $\begin{array}{l}\text { Sparitic, fine-grained, homogeneous anthraconite, } \\
\text { immediately below Andrarum Fm. .............. }\end{array}$ & 82 & -4.99 & -9.29 \\
\hline A-7 & M.Camb. & Borregård, Øleå & $\begin{array}{l}\text { Sparitic, fine- to medium grained anthraconite, } \\
\text { immediately below Andrarum Fm. ........... }\end{array}$ & 86 & -6.15 & -11.21 \\
\hline A-8 & M.Camb. & Borregård, Øleå & $\begin{array}{l}\text { Sparitic, medium-grained anthraconite, } \\
\text { Ptychagnostus punctuosus zone } \ldots \ldots \ldots \ldots \ldots \ldots \ldots\end{array}$ & 85 & -5.00 & -11.80 \\
\hline A-9 & U.Camb. & Læså, loose & $\begin{array}{l}\text { Microsparitic, laminated anthraconite, } \\
\text { Agnostus pisiformis zone............ }\end{array}$ & 78 & -6.45 & -11.32 \\
\hline A-10 & U.Camb. & South of Borregd., Øleå & $\begin{array}{l}\text { Sparitic, coarse-grained anthraconite, } \\
\text { top of Agnostus pisiformis zone....... }\end{array}$ & 78 & -8.61 & -9.20 \\
\hline A-11 & U.Camb. & South of Borregd., Øleå & $\begin{array}{l}\text { Sparitic, fine-grained anthraconite, } \\
\text { top of Agnostus pisiformis zone ................ }\end{array}$ & 82 & -6.25 & -12.22 \\
\hline A-12 & U.Camb. & South of Borregd., Øleå & $\begin{array}{l}\text { Sparitic, fine-grained anthraconite, } \\
\text { bottom of Olenus zone........... }\end{array}$ & 83 & -6.08 & -12.51 \\
\hline$A-13$ & U.Camb. & South of Borregd., Øleå & $\begin{array}{l}\text { Microsparitic, laminated anthraconite, } \\
\text { bottom of Olenus zone, average* } \ldots . .\end{array}$ & 82 & -5.52 & -11.78 \\
\hline $\begin{array}{l}A-14 \\
A-15\end{array}$ & $\begin{array}{l}\text { U.Camb. } \\
\text { U.Camb. }\end{array}$ & $\begin{array}{l}\text { Lxså, loose } \\
\text { South of Kalby, Læså }\end{array}$ & $\begin{array}{l}\text { Microsparitic, laminated anthraconite, Olenus zone } \ldots \\
\text { Sparitic, fine- to medium-grained anthraconite, } \\
\text { top Olenus zone, average* } \ldots \ldots \ldots \ldots \ldots \ldots \ldots \ldots \ldots \ldots\end{array}$ & 71 & $\begin{array}{l}-9.92 \\
-7.78\end{array}$ & $\begin{array}{l}-11.21 \\
-10.04\end{array}$ \\
\hline A-16 & U.Camb. & Brogård, Øleå & $\begin{array}{l}\text { Sparitic, medium-grained anthraconitic layer, } \\
\text { fossiliferous, Orusia lenticularis zone ........ }\end{array}$ & 61 & -9.98 & -12.00 \\
\hline $\begin{array}{l}\text { A-17 } \\
\text { A-18 }\end{array}$ & $\begin{array}{l}\text { U.Camb. } \\
\text { U.Camb. }\end{array}$ & $\begin{array}{l}\text { Vasegård, Læså } \\
\text { Vasegård, Læså }\end{array}$ & $\begin{array}{l}\text { Sparitic, fine-grained anthraconite, Leptoplastus zone.. } \\
\text { Sparitic, medium-grained anthraconite, Leptoplastus }\end{array}$ & 79 & -10.81 & -11.02 \\
\hline A-19 & U.Camb. & Vasegård, Lxså & 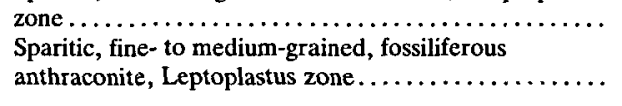 & 81 & $\begin{array}{l}-7.57 \\
-7.50\end{array}$ & $\begin{array}{l}-10.11 \\
-11.92\end{array}$ \\
\hline A-20 & U.Camb. & Vasegård, Lxså & $\begin{array}{l}\text { Sparitic, fine-grained fragment of anthraconite, } \\
\text { Protopeltura praecursor zone } \ldots \ldots \ldots \ldots \ldots \ldots \ldots \ldots\end{array}$ & 84 & -6.77 & -9.88 \\
\hline A-21 & U.Camb. & Vasegård, Læså, loose & $\begin{array}{l}\text { Sparitic, fine-grained anthraconite, } \\
\text { Protopeltura praecursor zone ...... }\end{array}$ & 75 & -10.75 & -11.90 \\
\hline
\end{tabular}

*average of two or more representative samples (excluding marginal and weathered material) of the same anthraconite, see Table 4. 


\begin{tabular}{|c|c|c|c|c|c|c|}
\hline Sample & Age & Locality & Description & 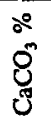 & $\begin{array}{l}x^{8} \\
0 \\
0\end{array}$ & $\begin{array}{l}x^{8} \\
0 \\
\infty \\
\infty\end{array}$ \\
\hline A-22 & U.Camb. & Vasegård, Læså, loose & $\begin{array}{l}\text { Sparitic, medium-grained, laminated anthraconite, } \\
\text { Peltura minor zone } \ldots \ldots \ldots \ldots \ldots \ldots \ldots \ldots \ldots\end{array}$ & 76 & -10.01 & -11.63 \\
\hline A-23 & U.Camb. & Vasegård, Læså, loose & $\begin{array}{l}\text { Sparitic, fine- to medium-grained laminated anthraco- } \\
\text { nite, Peltura minor zone } \ldots \ldots \ldots \ldots \ldots \ldots \ldots \ldots \ldots\end{array}$ & 69 & -11.13 & -11.63 \\
\hline $\begin{array}{l}\text { A-24 } \\
\text { A-25 }\end{array}$ & $\begin{array}{l}\text { U.Camb. } \\
\text { U.Camb. }\end{array}$ & $\begin{array}{l}\text { Vasegård, Læså, loose } \\
\text { Vasegård, Læså, loose }\end{array}$ & $\begin{array}{l}\text { Sparitic, fine-grained anthraconite, Peltura minor zone. } \\
\text { Sparitic, fine-grained anthraconite, } \\
\text { Peltura scarabaeoides zone } \ldots \ldots \ldots \ldots \ldots \ldots \ldots \ldots \ldots\end{array}$ & 89 & $\begin{array}{l}-9.81 \\
-6.30\end{array}$ & $\begin{array}{l}-11.23 \\
-10.81\end{array}$ \\
\hline A-26 & U.Camb. & Vasegård, Lxesả & $\begin{array}{l}\text { Sparitic, medium-grained anthraconite with cone-in- } \\
\text { cones, Peltura scarabaeoides zone } \ldots \ldots \ldots \ldots \ldots \ldots \ldots\end{array}$ & 73 & -10.78 & -12.01 \\
\hline A-27 & U.Camb. & Vasegård, Lxså & $\begin{array}{l}\text { Sparitic, coarse-grained anthraconite with cone-in-cones, } \\
\text { Peltura scarabaeoides zone } \ldots \ldots \ldots \ldots \ldots \ldots \ldots \ldots \ldots\end{array}$ & 86 & -8.25 & -10.75 \\
\hline A-28 & U.Camb. & Vasegård, Lxaså, loose & $\begin{array}{l}\text { Sparitic, fine-grained anthraconite, } \\
\text { Peltura scarabaeoides zone } \ldots \ldots \ldots \ldots \ldots \ldots \ldots \ldots\end{array}$ & 82 & -7.84 & -11.61 \\
\hline A-29 & U.Ordv. & Risebæk & $\begin{array}{l}\text { Microsparitic, homogeneous anthraconite, } \\
\text { Upper Dicellograptus Shale.............. }\end{array}$ & 68 & -10.51 & -10.81 \\
\hline \multicolumn{7}{|c|}{ Late diagenetic carbonates } \\
\hline S-1 & L.Camb. & Broens Odde, Snogebæk & $\begin{array}{l}\text { Carbonate cement from glauconitic siltstone, } \\
\text { Broens Odde member } . \ldots \ldots \ldots \ldots \ldots \ldots \ldots \ldots \ldots \ldots\end{array}$ & 13 & -6.47 & -14.83 \\
\hline S-2 & L.Camb. & Borregård, Øleå & 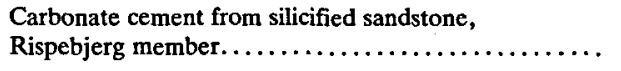 & 52 & -9.65 & -11.80 \\
\hline S-3 & M.Camb. & Borregård, Øleå & $\begin{array}{l}\text { Sparite, from cavity inside shell, Exsulans } \\
\text { Limestone Fm............................. }\end{array}$ & 97 & -0.59 & -7.41 \\
\hline $\begin{array}{l}S-4 \\
S-5\end{array}$ & $\begin{array}{l}\text { M.Camb. } \\
\text { L.Ordv. }\end{array}$ & $\begin{array}{l}\text { Borregård, Øleå } \\
\text { Limensgade, Laså }\end{array}$ & 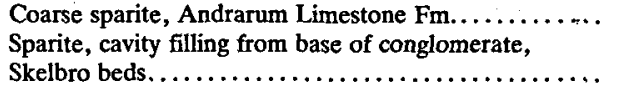 & 95 & $\begin{array}{l}-9.32 \\
-4.40\end{array}$ & $\begin{array}{l}-8.15 \\
-3.90\end{array}$ \\
\hline S-6 & L.Ordv. & Limensgade, Laså & $\begin{array}{l}\text { Sparite, fissure filling, } 5 \mathrm{~cm} \text { below top of } \\
\text { conglomerate, Skelbro beds } \ldots \ldots \ldots \ldots \ldots \ldots \ldots \ldots\end{array}$ & 31 & -1.47 & -8.42 \\
\hline S-7 & L.Ordv. & Skelbro & $\begin{array}{l}\text { Sparitic from vein, } 5 \mathrm{~mm} \text { thick, } 210 \mathrm{~cm} \text { above to of } \\
\text { conglomerate, Komstad Limestone } \mathrm{Fm} . \ldots \ldots \ldots \ldots \ldots\end{array}$ & 95 & -21.84 & -4.30 \\
\hline S-8 & L.Ordv. & Skelbro & $\begin{array}{l}\text { Sparite from vein, } 25 \mathrm{~mm} \text { thick, } 210 \mathrm{~cm} \text { above top of } \\
\text { conglomerate, Komstad Limestone } \mathrm{Fm} . \ldots \ldots \ldots \ldots \ldots\end{array}$ & 93 & -14.92 & -7.06 \\
\hline S-9 & Recent & Skelbro & $\begin{array}{l}\text { Stalactitic carbonate from surface of limestone in } \\
\text { Skelbro quarry } \ldots \ldots \ldots \ldots \ldots \ldots \ldots \ldots \ldots \ldots \ldots \ldots\end{array}$ & 90 & -6.73 & -6.15 \\
\hline S-10 & U.Ordv. & Risebæk & Sparite from vein filling in anthraconite $A-29 \ldots \ldots \ldots$ & 96 & -11.03 & -6.25 \\
\hline
\end{tabular}

Table 2. Average and range for isotopic composition of investigated samples

\begin{tabular}{|c|c|c|c|c|c|c|}
\hline \multirow{2}{*}{ Unit } & \multicolumn{3}{|c|}{ Average } & \multirow[t]{2}{*}{$\mathbf{n}$} & \multicolumn{2}{|c|}{ Range } \\
\hline & ${ }^{13} \mathrm{C} \%$ & & ${ }^{18} \mathrm{O} \%$ & & ${ }^{13} \mathrm{C} \%$ & ${ }^{18} \mathrm{O} \%$ \\
\hline Exsulans Imst. & -1.12 & & -9.29 & 4 & 2.07 & 2.98 \\
\hline Kalby Clay & -2.34 & & -8.57 & 4 & 1.73 & 2.98 \\
\hline Andrarum Imst. & -2.06 & & -11.38 & 4 & 1.12 & 1.11 \\
\hline Komstad Imst. s.s. & -0.81 & & -11.38 & 12 & 0.97 & 1.60 \\
\hline Skelbro beds & -3.50 & & -11.34 & 3 & 1.16 & 0.71 \\
\hline Jerrestad Fm. & -5.21 & & -11.07 & 3 & 0.23 & 0.40 \\
\hline Anthraconite, M.C. & -4.97 & & -10.63 & 8 & 2.73 & 3.17 \\
\hline Anthraconite, U.C. & -8.40 & & -11.24 & 20 & 5.61 & 3.31 \\
\hline Anthraconite, U.O. & -10.51 & & -10.81 & 1 & - & - \\
\hline
\end{tabular}


Table 3. Effect of Weathering

\begin{tabular}{llrr}
\hline Sample & Description & $\delta^{13} \mathrm{C} \%$ & $\delta^{18} \mathrm{O} \%$ \\
\hline E-1 to E-4 & Exsulans Imst. not affected by major weathering & -1.12 & -9.29 \\
E-5 to E-8 & Kalby Clay, weathered residue of Exsulans lmst. & -2.34 & -8.57 \\
K-10 to K-15 & Komstad Imst. Vasagård I core, fresh & -0.72 & -11.66 \\
K-4 to K-9 & Komstad Imst., Skelbro quarry, fresh & -0.90 & -11.30 \\
K-16 & Komstad lmst., Skelbro quarry, exposed surface & -1.93 & -10.79 \\
K-17 & Komstad Imst., top of unit in Vasagărd I core & -0.39 & -8.80 \\
S-9 & Stalactitic crust from surface, Skelbro quarry & -6.73 & -6.15 \\
A-15,1 & Anthraconite, weathered surface & -11.53 & -9.35 \\
A-15,4 & Anthraconite, 7.8 cm below surface & -7.80 & -9.92 \\
\hline
\end{tabular}

pleted Lower Palaeozoic limestones and local ground water which has $\delta^{18} \mathrm{O}$-values around -8 $\%$. A reversed effect is observed for the carbon isotope composition, which probably reflects a local ${ }^{13} \mathrm{C}$-depleted source of dissolved ground water carbon.

A similar but much smaller effect is found in the Komstad Limestone unit. Comparison between the core samples and the surface-exposed quarry samples reveals an ${ }^{18} \mathrm{O}$-enrichment of 0.4 $\%$ for the latter (see fig. 3 ). The similar difference in ${ }^{13} \mathrm{C}$ is less than $-0.2 \%$. The topmost samples from the quarry and the core, which both show signs of weathering, are significantly en-

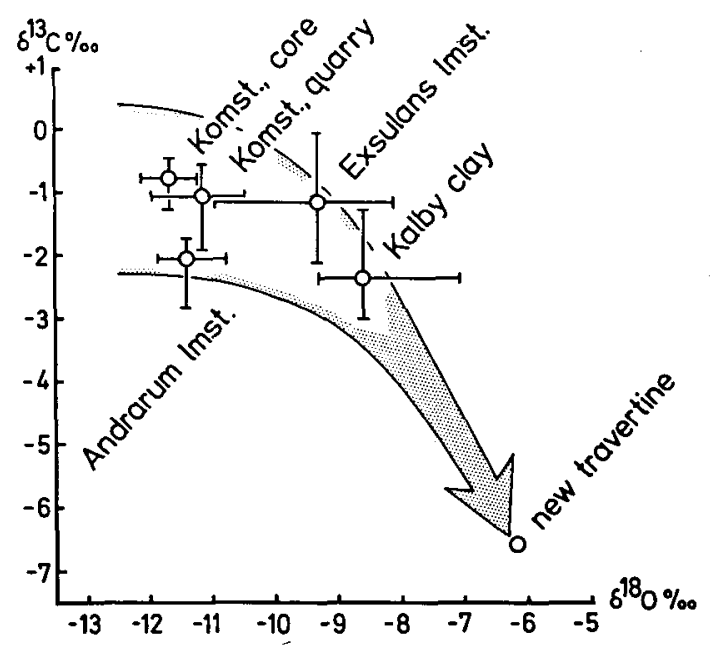

Figure 3. Effect of weathering on the Cambro-Ordovician limestone of Bornholm. The figure shoes average values and spread of the individual units. Exsulans limestone and Kalby Clay exhibit visual signs of weathering. The travertine originates from the surface of the Komstad limstone in the Skelbro Quarry. riched in ${ }^{18} \mathrm{O}$ as compared to the rest of the Komstad material. The ${ }^{13} \mathrm{C}$-data are not conclusive.

Weathered surface samples of anthraconitic concretions are enriched in ${ }^{18} \mathrm{O}$ as compared to their central parts. The corresponding ${ }^{13} \mathrm{C}$-data are variable, probably reflecting different sources for the exchanging carbon.

The overall effect of isotopic exchange related to weathering seems to be enrichment in ${ }^{18} \mathrm{O}$ and depletion in ${ }^{13} \mathrm{C}$ of the affected carbonates. This is in accordance with the observed isotopic composition of recent stalactitic crusts formed on the exposed surface of the Skelbro Quarry limestone (sample S-9). The effects, however, are small, and most of the investigated samples have not suffered from exchange processes due to weathcring.

Intra-unit variation

Intra-concretionary variation has been determined for 4 anthraconites (table 4). Excluding weathered surface samples the $\delta^{13} \mathrm{C}$-variability is small $(<1.3 \%$ ) for each concretion, and no welldefined zoning can be identified. Intra-concretionary variations are larger for the oxygen isotope ratios (up to $2.5 \%$ ), but only in relation to differences between surface-near samples and the more central parts. Moreover, no trend is found in the data. Therefore, the sampling procedure for the anthraconites can be considered representative.

Intra-unit variation in the primary limestone material (table 2) is relatively large for the Exsulans Limestone Fm., which lithologically is the most heterogeneous of the units. Intra-unit variation in the rest of the material is small, and the 
Table 4. Intraconcretionary variations of selected anthraconites.

\begin{tabular}{|c|c|c|c|c|c|}
\hline Sample & $\begin{array}{l}\text { Location in } \\
\text { concretion }\end{array}$ & Description & $\mathrm{CaCO}_{3} \%$ & $\delta^{13} \mathrm{C} \%$ & $\delta^{18} \mathrm{O} \%$ \\
\hline$A-4,1$ & $1.5 \mathrm{~cm}$ below surface & Sparitic, medium-grained, black & 82 & -3.35 & -11.07 \\
\hline$A-4,2$ & $3.0 \mathrm{~cm}$ below surface & Sparitic, coarse-grained with cone-in-cone structure* & 86 & -2.89 & -9.25 \\
\hline$A-4,3$ & $6.5 \mathrm{~cm}$ below surface & Sparitic, fine-grained, black* & 84 & -3.99 & -8.69 \\
\hline$A-3,1$ & $0.5 \mathrm{~cm}$ below surface & Sparitic, coarse-grained with cone-in-cone structures & 89 & -3.97 & -8.67 \\
\hline$A-3,2$ & $1.2 \mathrm{~cm}$ below surface & Sparitic, medium-grained, black & 83 & -4.81 & -11.23 \\
\hline$A-3,3$ & $2.5 \mathrm{~cm}$ below surface & Sparitic, coarse-grained with cone-in-cone structures* & 87 & -4.80 & -10.32 \\
\hline$A-3,4$ & $3.5 \mathrm{~cm}$ below surface & Sparitic, fine-grained, black* & 90 & -5.07 & -10.37 \\
\hline$A-3,5$ & $4.8 \mathrm{~cm}$ below surface & Sparitic, fine-grained, black & 87 & -5.24 & -10.70 \\
\hline$A-13,1$ & $1.0 \mathrm{~cm}$ below surface & Sparitic, fine-grained, black* & 81 & -5.67 & -12.60 \\
\hline A-13,2 & $3.6 \mathrm{~cm}$ below surface & Microsparitic, black* & 85 & -5.62 & -12.20 \\
\hline A-13,3 & $5.8 \mathrm{~cm}$ below surface & Microsparitic, black* & 80 & -5.24 & -11.81 \\
\hline A-13,4 & $8.5 \mathrm{~cm}$ below surface & Sparitic, fine-grained & 83 & -5.29 & -11.73 \\
\hline A-15,1 & $0.2 \mathrm{~cm}$ below surface & Sparitic, fine-grained, weathered & 83 & -11.53 & -9.35 \\
\hline A-15,2 & $1.8 \mathrm{~cm}$ below surface & Sparitic, fine-grained, partly weathered & 80 & -9.64 & -10.26 \\
\hline A-15,3 & $4.5 \mathrm{~cm}$ below surface & Sparitic, fine-grained, black* & 84 & -7.78 & -10.15 \\
\hline A-15,4 & $7.8 \mathrm{~cm}$ below surface & Sparitic, fine-granied, black* & 83 & -7.80 & -9.92 \\
\hline
\end{tabular}

* Denotes samples used for calculation of average concretionary values given in Table 2.

average values for each unit can be considered as representative.

\section{Variations related to lithology}

The isotopic composition of the individual units is shown in fig. 4. The most conspicuous difference is found between the carbon isotope ratios of primary limestones and anthraconites, which in their turn can be divided into Middle Cambrian and Upper Cambrian to Ordovician groups. The calcite-cemented Jerrestad mudstone horizon has a carbon isotopic composition comparable to the anthraconites, while the two Silurian samples are enriched in ${ }^{13} \mathrm{C}$ to a degree comparable to the primary limestones. Within the primary limestone group carbon isotope differences are related to weathering effects (Kalby Clay and Exsulans Limestone), and to minor lithological differences within the Andrarum Limestone and Skelbro beds as compared to the post Skelbro bed part of the Komstad Limestone. The late diagenetic carbonates have $\delta^{13} \mathrm{C}$-values similar to or more negative than the anthraconites. Most significant ${ }^{13} \mathrm{C}$-depletion is found in the vein sparites S-7 and S-8, which must have formed from a carbon source totally different from the Komstad material

Differences in oxygen isotope composition between the lithological units are small and insig- nificant except for the late diagenetic carbonates, most of which are markedly enriched in ${ }^{18} \mathrm{O}$. It is notable, however, that the carbonate cement of the Lower Cambrian sandstone samples (S-1 and $\mathrm{S}-2$ ) have an oxygen isotope composition close to that of the primary and early diagenetic carbonates. The minor ${ }^{18} \mathrm{O}$-enrichment of the Exsulans Limestone material and the Middle Silurian sample as compared to the rest of the primary material is probably related to surface exchange processes (weathering).

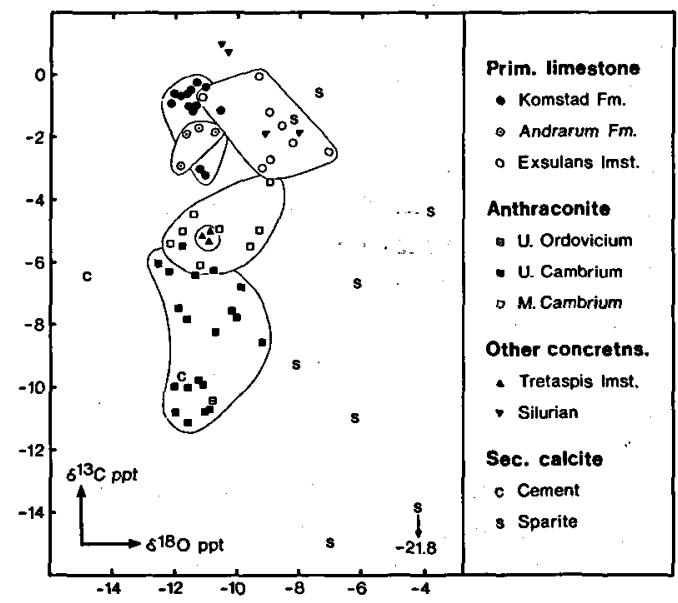

Figure 4. Carbon and oxygen istotope compostion of investigated calcareous material from the Lower Palaeozoic deposits of Bornholm. 
Variations related to stratigraphy

In fig. 5 , the carbon and oxygen isotope compostion of the investigated material is plotted against the stratigraphical position. Minor fluctuations in $\delta^{18} \mathrm{O}$-values are seen in the Middle Cambrian part of the profile. These fluctuations probably reflect weathering and/or ground water exchange effects affecting the thin-bedded Exsulans Limestone and some of the related anthraconites. For the rest of the profile the oxygen isotope composition is remarkably uniform.

The carbon isotope values have a more pronounced stratigraphical variation. Closer inspection reveals most of these fluctuations to be related to lithology: the already described differences between primary limestones and concretions. Considered separately, no significant stratigraphical trends are found in the primary limestone composition. The anthraconites, on the other hand, exhibit a gradual ${ }^{13} \mathrm{C}$-depletion from the Middle Cambrian to the Upper Ordovician. Of the late diagenetic carbonates, the calcite-cemented Lower Cambrian sand- and siltstones (S-1 and S-2) have a carbon isotope composition similar to that of the anthraconites.

\section{Interpretation of results}

For a carbonate rock, the initial stable isotope composition is given by temperature, oxygen isotope composition of water and carbon isotope compostion of bicarbonate ions. The composition is subject to changes by diagenetical re-equilibration processes ranging from early diagenetic, pre-compactional modifications over burial-diagenetic alterations at elevated temperatures to weathering. Any attempt to estimate primary, palaeo-environmental parameters from the isotopic composition of a diageneticaly modified limestone therefore implies an analysis of the history and magnitude of these modifications. On the other hand, isotopic studies of diagenetically altered limestones can often be applied as a tool for the evaluation of the history of diagenesis.

In the present case we are dealing with rocks of considerable age. Therefore, any interpretation of data must take into account both the isotopic evolution of ocean water in Phanerozoic time and diagenetic modifications of the rocks. The fol- lowing approach is based on a set of semiquantitative models that discuss the expected isotopic composition of Lower Palaeozoic carbonates on the basis of certain assumptions about depositional parameters and diagenetic history.

The character of the geological setting suggests the consideration of three different models: one model depicting a rock suite unaffected by diagenetic modifications, another model describing material affected by early diagenetic recrystallization in subaerial to phreatic environments and a third model dealing with carbonates modified by burial diagenesis at elevated temperatures. Hydrothermal modifications from surface-near intrusive bodies as described by Taylor (1977) have not been considered here, as geological evidence contradicts this type of influence. The effects of late diagenetic, meteoric water exchange processes (weathering) have been demonstrated to be minimal and are not considered either. In the following discussion each of the three models will be examined and the expected isotopic distributions will be compared to the observed data.

\section{Model 1. No diagenetic modifications}

Oxygen isotope composition of calcium carbonate precipitated under equilibrium conditions is given by the relationship (O'Neill et al. 1969):

$$
\begin{aligned}
& 10^{3} \ln \alpha\left(\mathrm{CaCO}_{3}-\mathrm{H}_{2} \mathrm{O}\right)= \\
& 2.78\left(10^{5} / \mathrm{T}^{2}\right)-2.89
\end{aligned}
$$

where $\alpha$ is the fractionation factor for calcium carbonate and water, and $\mathrm{T}$ is the temperature in ${ }^{\circ} \mathrm{K}$.

The original isotopic composition of Lower Palaeozoic carbonates can be calculated from (1), provided reasonable estimates of $T$ and oxygen isotope composition of water are established.

The knowledge of Lower Palaeozoic climates of Scandinavia is scanty and the palaeontological, sedimentological and geophysical evidence is subject to much debate (for review, see Spjeldnæs 1981). Cold water conditions are indicated in the late Eocambrian by the Moelv Tillites in Norway (Oftedahl 1945 and Bjørlykke 1966), and warm water conditions by the presence of bahamitic type carbonate sediments and reef buildups (Oslo, Siljan) in the Late Caradoc and Ashgill of the Ordovician (Webby 1984) and 

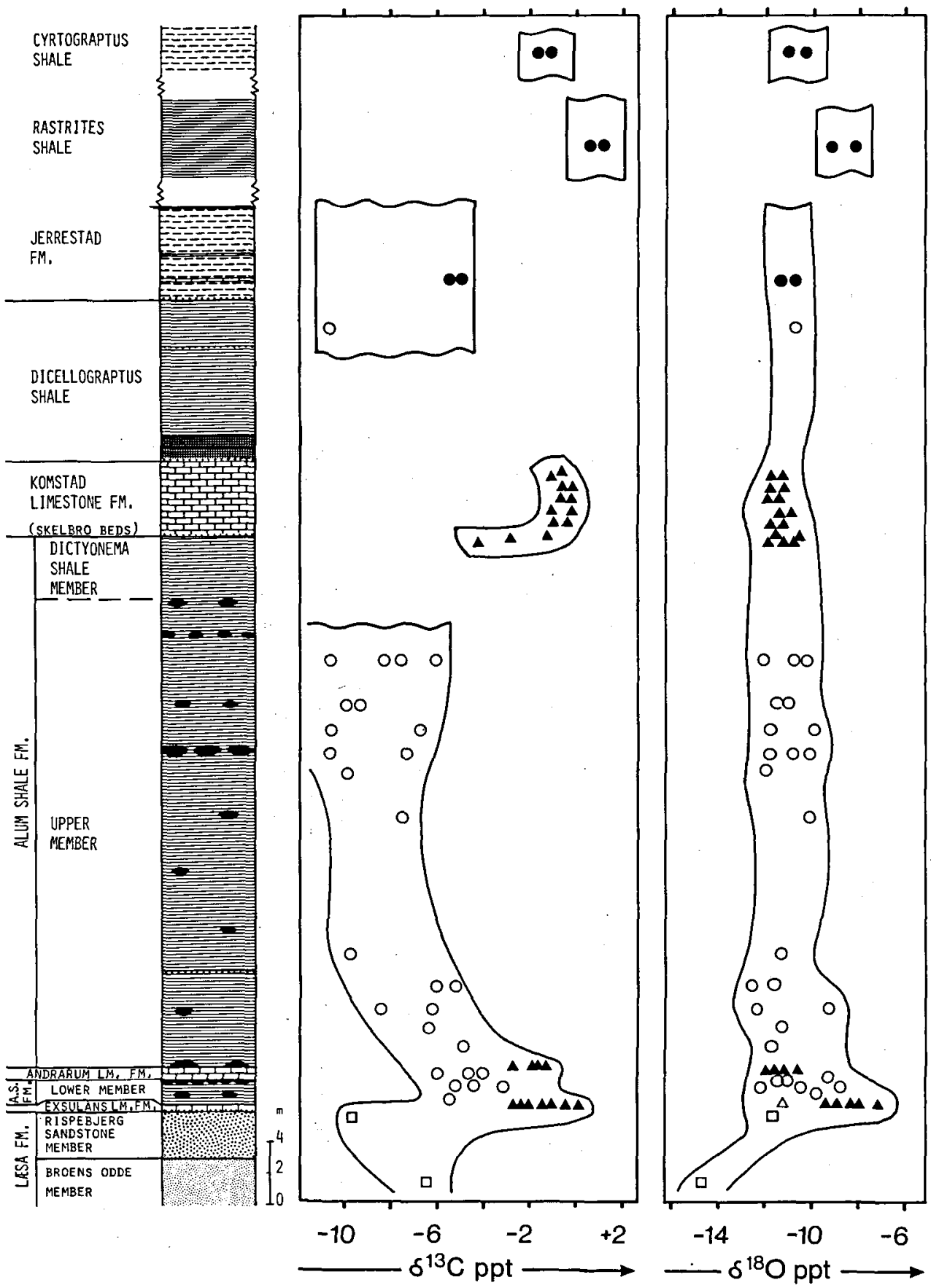

A prim. limestone; o anthraconite; $\bullet$ other concr. limest.; व sec. calcite

Figure 5. Stratigraphical variability in carbon and oxygen isotope composition of the investigated calcareous material from Bornholm. Chronostratigraphical subdivisions are given in figure 1. 
the Wenlock and Ludlow of the Silurian (Gotland, Oslo (Spjeldnæs 1981)). Following these pieces of evidence, an estimated temperature range for the Southern Scandinavian Lower Palaeozoic ocean from $0^{\circ}$ to $25^{\circ} \mathrm{C}$ seems realistic.

An estimate of the isotopic composition of the precipitating water phase must be based on the assumption of a fully marine environment (see above), where the oxygen isotope composition of local waters is identical to that of the Lower Palaeozoic ocean in general. Unfortunately, no reliable data exist for the oxygen isotope composition of pre-Carboniferous oceans, and one has to rely on indirect lines of evidence. Two contrasting opinions have been published about the oxygen isotopic evolution of the world oceans. Perry (1967) and Walls et al. (1977) argued that the oceans have experienced a gradual enrichment in ${ }^{18} \mathrm{O}$ frem Early Precambrian time till now. According to this concept the Lower Palaeozoic oceans were depleted in ${ }^{18} \mathrm{O}$ by 2 to $3 \%$ as compared to modern oceans. On the other hand, Taylor (1977) and Brand (1982) suggest a constant ${ }^{18} \mathrm{O}$-composition of ocean water identical to the pre-glaciation Cenozoic values $(-1 \%$, Shackleton and Kennett 1975) back into Precambrian and Carboniferous time respectively. A more extreme interpretation is given by Lindström (1984), who argues for an Ordovician sea water composition 5 to $6 \%$ lighter than today. Reliable limits for the $\delta^{18} \mathrm{O}$-values of the Lower Palaeozoic ocean are here considered to be from $-3 \%$ to $0 \%$ SMOW.

An estimated original oxygen isotope composition can now be calculated for Lower Palaeozoic carbonates and early diagenetic concretions of marine origin. Based on the temperature and $\delta^{18} \mathrm{O}_{\text {ocean }}$-ranges given above, the carbonate $\delta^{18} \mathrm{O}$ values will fall within the interval from $-4 \%$ to $\mathrm{O} \% \mathrm{PDB}$ (see fig. 6). Measured $\delta^{18} \mathrm{O}$-values for the investigated, non-weathered carbonates are from $-12 \%$ to $-9 \%$ PDB. The difference between the calculated and the measured values is considered significant. Consequently, the oxygen isotope composition of the investigated carbonates cannot reflect original depositional parameters.

The carbon isotope composition of calcium carbonate precipitated directly from sea water is a function of the ${ }^{13} \mathrm{C} /{ }^{12} \mathrm{C}$-ratio of the dissolved inorganic carbon reservoir and of temperature.
The present isotopic composition of sea water bicarbonate ions is approximately $+2 \%$, corresponding to a primary carbonate compostion of $0 \%$ to $+4 \%$ (Mook 1971). This range, which is related to the distribution of carbon between the organic and the inorganic carbon reservoirs, is believed not to have changed significantly during the last 2000 ma years (Keith \& Weber 1964, Schidlowski et al. 1975). Minor secular variations in the $\delta^{13} \mathrm{C}$-values of marine carbonates have been reported, however (Veizer \& Hoefs 1976, Veizer et al. 1980), and most reliable estimates of Cambro-Ordovician $\delta^{13} \mathrm{C}$ carbonate-values are between $-1 \%$ and $+2 \%$ ( $-0.57 \%$ $\pm 0.17 \%$, $n=$ 75 , Veizer et al. 1980).

Considering the expected carbon isotope composition of concretions, another line of arguments has to be followed. Early diagenetic concretions precipitate from pore waters with a carbon isotope composition different from that of the sea water. The main source of concretionary carbonate carbon is $\mathrm{CO}_{2}$ or $\mathrm{HCO}_{3}^{-}$formed by bacterial decomposition of sedimentary organic matter (Berner, 1968 and Raiswell, 1976). The type of bacterial process is related to depth below sea bottom, sulphate reduction being dominant in the upper few metres giving way to fermentation at greater depths.

According to the model by Irwin \& Curtis (1977) sulphate reduction of organic material is not accompanied by any noticeable fractionation of carbon isotopes. Calcareous concretions formed in the bottom zone dominated by sulphate reduction will thus have a carbon isotope composition similar to that of the sedimentary organic material (normally less than $-15 \%$ ). Fermentation, on the other hand, is charcterized by a large fractionation of the carbon isotopes in the resulting methane and carbon dioxide, the latter being strongly enriched in ${ }^{13} \mathrm{C}$ compared to the organic material. Concretions grown preferentially from $\mathrm{CO}_{2}$ formed by this process will have $\delta^{13} \mathrm{C}$-values in the positive range (normally from $0 \%$ to $+5 \%$ ). Normally, early diagenetic concretions form in both bottom zones, and any isotopic composition between the two extremes mentioned above is possible.

In the present case, the relatively high concentration of pyrite in the alum shales (up to $14 \%$, Hansen 1945) points to at least part of the concretions being formed in the zone of sulphate 

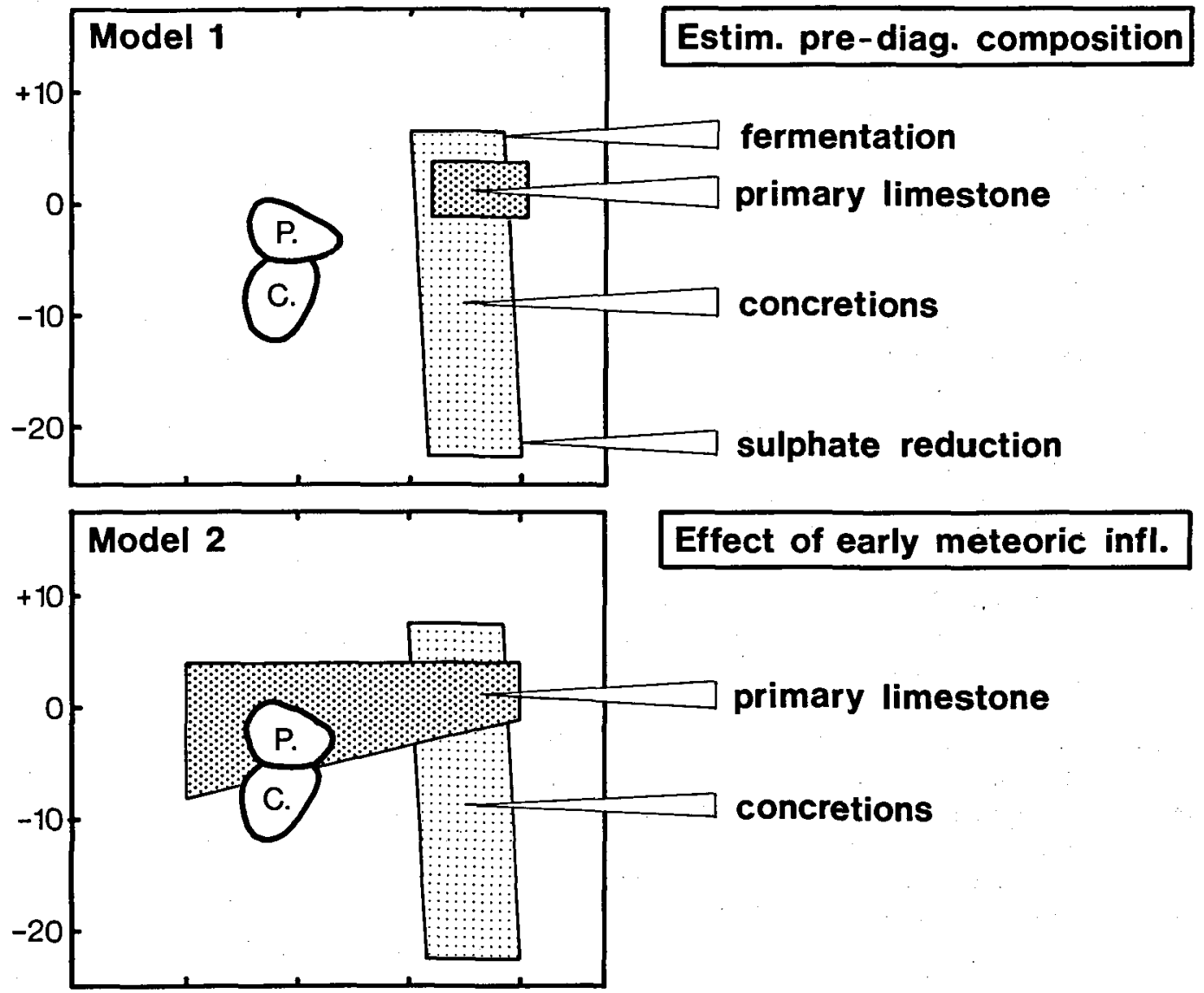

primary limestone

concretions

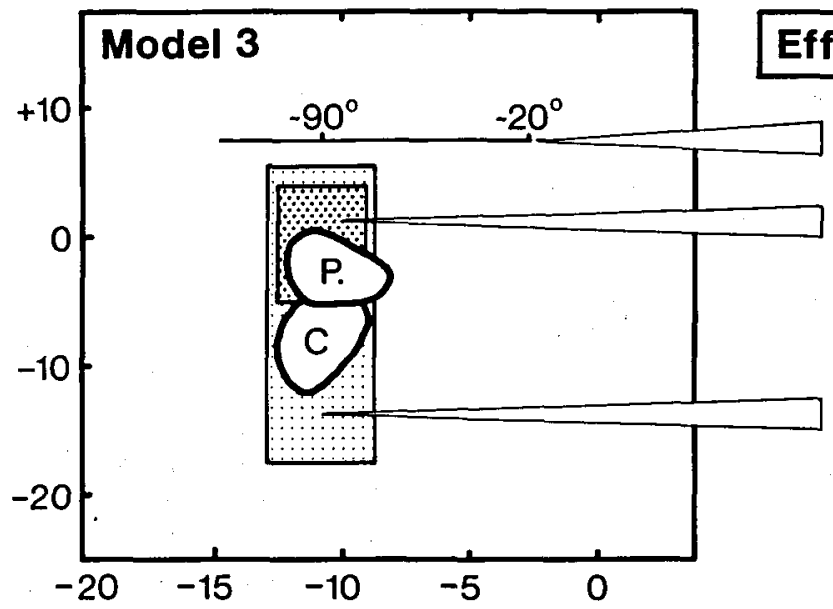

\section{concretions}

$\delta^{13} \mathrm{C}$ ppt rel. PDB

Effect of burial diagenesis

estimated burial temp.

primary limestone

Figure 6. Measured compositions of the investigated Cambro-Silurian calcareous units compared to estimated compositions calculated from different prerequisites. Further explanation in the text. 
reduction. Buchardt \& Cederberg (in prep.) report $\delta^{13} \mathrm{C}$-values of kerogen from the investigated sections between $-30 \%$ and $-28 \%$, which can be taken as a rough estimate of the carbon isotope composition of the original organic phase. Carbon isotope compositions of the sudied anthraconites can be expected to range from these values to a pure fermentation composition of about $+5 \%$.

The investigated carbonates have a carbon isotope distribution, the trends of which are in accordance with the expected values (fig. 6). Minor deviations between actual and estimated values for some of the primary carbonates do, however, indicate postdepositional modifications to a certain degree. The most typical "sea water equilibrium" values are found in the thickest primary units (the Komstad Fm.), while the Exsulans Limestone, the Andrarum Limestone and the marginal parts of the Komstad Fm. are slightly lighter than expected.

The anthraconite undoubtedly reflect decomposition of organic matter in the bottom sediments, but none of the samples have a pure "sulphate reduction composition". Lack of zoning in isotopic composition may indicate diagenetic intra-concretionary homogenization to a certain degree.

In summary, both the carbon and the oxygen isotope compositions of the investigated carbonates deviate from the expected values and consequently must have suffered from postdepositional modifications. These processes caused total obliteration of original oxygen compositions and minor changes in the carbon isotope distribution. It is evident, therefore, that the discussed model does not satisfactorily explain the observed data.

Model 2. Early diagenetic modifications in a meteoric water environment

Early diagenetic modifications caused by re-equilibrating proceses between marine limestone and meteoric water have been described from areas where carbonates are exposed subaerially. Petrographically, these modifications are characterized by recrystallization of metastable minerals, formation of secondary porosity and development of karst sturctures. Several authors have explained the occurrence of discontinuity horizons in the Komstad Limestone as a result of subaerial exposure (Grönwall 1899; C. Poulsen 1936, V. Poulsen 1966). However, these structures may have formed synsedimentarily as subaquaceous lithifications. Other evidence for subaerial exposure are not conclusive.

The degree of isotopic modification of a carbonate rock exposed to meteoric water is related to the extent of lithification of the carbonate (e.g. Gross 1964, Allan \& Matthews 1982). An unstable, highly porous and permeable carbonate sediment is susceptible to major modification in isotope chemistry. A thoroughly lithified carbonate rock having low permeability will suffer only superficial modification at the reaction rates given by surface temperatures. The latter type of effects has already been referred to as results of weathering, and the discussion can be limited to processes taking place in an early diagenetic, meteoric water environment.

Of the carbonates under study, only the primary limestone of the Exsulans, Andrarum and Komstad Fms. had prelithification properties suitable for isotopic exchange with meteoric water. The concretions lithified during growth and therefore were little susceptible to isotopic exchange. Moreover, they were protected by a highly impermeable package of organic-rich clays. Any search for traces of meteoric water exchange processes can therefore be limited to the primary limestone units.

Meteoric water today generally is depleted in ${ }^{18} \mathrm{O}$ as compared with ocean water. The difference reflects the isotopic fractionation in the atmospheric circulation systems which is related to distance from equator (Dansgaard 1964). Similar fractionation mechanisms must have been active during most of global history. Palaeo-latitudes of the studied area during the Early Palaeozoic were from $20^{\circ}$ to $60^{\circ} \mathrm{N}$ (e.g. Cocks \& Fortey 1982) and meteoric water of the region possibly was depleted in ${ }^{18} \mathrm{O}$ by $5 \%$ to $10 \%$.

Recent meteoric waters show a large range in ${ }^{13} \mathrm{C}$-composition of the dissolved inorganic carbon. Most positive $\delta^{13} \mathrm{C}$-values are found in water equilibrated with atmospheric carbon dioxide, while soil water, ground water and river water normally are depleted in ${ }^{13} \mathrm{C}$ with as much as 20 $\%$ owing to contributions of ${ }^{13} \mathrm{C}$-low carbon from decomposing terrestrial organic matter (Degens 1969, Deines 1980). This effect causes most me- 
teoric water-influenced limestones to be markedly depleted in ${ }^{13} \mathrm{C}$ as compared to original, marine limestones (Allan \& Matthews 1982). An extrapolation of this trend back to the Early Palaeozoic is rendered difficult by lack of knowledge of the type and extent of terrestrial plant life at that time. However, the nonexistence of higher vascular plant life does not exclude any occurrence of e.g. fresh water algal communities or certain types of true terrestrial algal "mats". A ${ }^{13} \mathrm{C}$-depleted carbon contribution to the Early Palaeozoic meteoric waters therefore cannot be excluded. A conservative estimate of the amount of organically derived carbon in the fresh water to one-fifth of present values leaves an approximate ${ }^{13} \mathrm{C}$-range of Early Palaeozoic meteoric water from about $-8 \%$ to $-3 \%$.

An estimate of isotopic distribution reflecting early diagenetic, meteoric water modifications of the investigated material must take these considerations into account. Thus, primary carbonates modified in meteoric water environments should exhibit $\delta^{18} \mathrm{O}$ and $\delta^{13} \mathrm{C}$-ranges from about $14 \%$ to $-2 \%$ and $-8 \%$ to $+3 \%$ respectively depending on degree of modification. The concretions, on the other hand, should have preserved their original values.

This distribution is not in accordance with the measured values (see fig. 6). Only the oxygen isotope composition of the primary limestone material can be interpreted as a result of meteoric water exchange processes at low temperatures. The other data do not fit into the model which - consequently - is not considered adequate. In conclusion, there is no convincing evidence for a meteoric water, early diagenetic alteration of the investigated carbonates.

Model 3. Burial diagenesis at elevated temperatures

During burial of a sedimentary rock suite isotopic exchange will take place between minerals, pore water and dissolved ions. The process is slow at surface temperatures but accelerates with increasing depth. The result is a general re-equilibration of isotopes between the reacting phases leading to overall homogenization of the isotopic compositions of the rocks. The magnitude of the effect depends on the ratio between concentrations of exchanging isotopes in the minerals and fluid (dissolved species/solid species) and on the size of change in isotopic fractionation with increasing temperature $(\mathrm{d} \delta / \mathrm{dT})$.

The most pronounced influence from burial diagenesis is to be expected for the oxygen isotope compositions. In a rock suite as that investigated here the initial water/rock ratio is large. Isotopic homogenization will be almost complete, and the decreasing fractionation between pore water and carbonate minerals will lead to changes in the minerals rather than in the water phase. The result will be a gradual depletion in ${ }^{18} \mathrm{O}$ of carbonate minerals in the range of $10 \%$ pr. $100^{\circ} \mathrm{C}$ increase in temperature $\left(\mathrm{d}_{\mathrm{CaCO}_{3}-\mathrm{H}_{2} \mathrm{O}} / \mathrm{dT}=\right.$ 0.0001). This ${ }^{18} \mathrm{O}$ depleting effect will be enhanced by influence from ${ }^{18} \mathrm{O}$-poor meteoric water introduced into the sedimentary pile from external sources. The effect is known from largescale hydrothermal convection systems initiated from intrusive magmatic bodies (Taylor 1977, Forester \& Taylor 1977), and from examples where high-permeable rock-units are "leaking" to near-surface meteoric water reservoirs (e.g. Magaritz 1974). The effect probably was of minimal importance in the present case.

Smaller effects are to be expected for the carbon isotope compositions. In most cases the ratio between dissolved carbon and solid carbonate carbon is small, and exchange processes will be less effective. Moreover, the change in fractionation with temperature is smaller than for oxygen. As a consequence, homogenization between minerals having different initial ${ }^{13} \mathrm{C}$ content will be less complete, and the general shift in carbon isotope composition of the carbonate rocks with increasing temperature will be small. Most extensive homogenizations are to be expected between close-lying carbonate units, while isolated carbonate rocks like concretionary bodies in shale sequences will preservec most of their original carbon isotope composition.

The presence of a water phase is crucial for the isotopic exchange processes. The gradual expulsion of pore water will lead to a decreasing extent of exchange at greater depths. At a certain depth the rock suite will have lost all its porosity, and further exchange will be limited to cracks and fissures in the otherwise non-porous rock. This depth represents a "closing temperature" recognizable from the oxygen isotope composition of the affected rocks. Later changes in diagenetic 
temperatures are not expected to induce major modifications in the isotopic composition of the carbonate rocks.

The observed data fit into this model (see fig. 6). The studied carbonates are close to total homogenization with regard to oxygen isotope composition, the only exceptions being the vein calcites, which probably postdate the homogenization "event". Moreover, the oxygen isotope ratios of all pre-vein carbonates are depleted in ${ }^{18} \mathrm{O}$ as compared to expected initial values. The magnitude of the depletion is dependent upon the estimation of the original depositional parameters, but range from 7 to $13 \%$.

The carbon isotope compositions of the investigated rocks are heterogeneous and seem to reflect original, pre-burial conditions. The only exceptions are the Middle Cambrian limestone units and anthraconites which probably have undergone local exchange and homogenization to a certain degree.

Considered together, these arguments favour burial diagenesis as the explanation to the observed data. Consequently, the third model is accepted here.

An estimated minimal temperature of loss of porosity can be calculated from the observed ${ }^{18} \mathrm{O}$ depletion of the carbonates. Acceptance of Taylor's hypothesis of a constant oxygen isotope composition of ocean water approximately equal to $-1 \%$ for the last billion years (Taylor 1977) leads to a closing temperature between $80^{\circ}$ and $90^{\circ} \mathrm{C}$ provided pore water was of pure marine origin. Influence of ${ }^{18} \mathrm{O}$-depleted meteoric water will result in the calculated closing temperatures being too low. Further modifications at higher temperatures can only be evaluated from mineral/ mineral equilibration reactions involving minerals other than calcite, e.g. clay minerals. These reactions have not been studied here, as equilibration is known first to be established at metamorphic temperatures (Eslinger \& Savin 1973).

\section{Testing of the proposed model}

Testing of the deep burial model can be achieved by evaluation of non-isotopical data confirming the high-temperature influence on the Lower Palaeozoic rocks of Bornholm.
Thermal evidences

Until recently little was known about the postdepositional, thermal history of the Lower Palaeozoic sequence of Bornholm. New data from studiues of vitrinite refelctance and clay mineralogy of the Alum Shale Fm. do indicate high temperature modifications of the rocks (Thomsen et al. 1983). Vitrinite reflectance values around $2 \% R_{o}$ correspond to a post-mature stage with respect to oil generation while clay mineralogy points to a low metamorphic grade. A corresponding conclusion can be reached from the conodont colour alteration index value of approx. 5 (Svend Stouge, pers. communication 1983).

Burial or magmatic heating?

Extensive magmatic activity is known to have taken place during the Carboniferous and Permian periods in Scania north of Bornholm (Klingspor 1976), where older rocks were transsected by numerous basaltic dykes. However, no dykes are reported to cut the Lower Palaeozoic of Bornholm, and a postulated Lower Palaeozoic age for one of the dykes cutting the older basement rocks in Northern Bornholm is not conclusive (Abrahamsen 1977). Moreover, no indications are found of any deep-seated intrusive body affecting the Palaeozoic rocks in any other way. Local magmatic heating can therefore be excluded as the explanation to the above described thermal impact.

\section{Non-thermal evidence}

Non-thermal arguments in favour of a deep burial diagenetic history are mostly geophysical. As mentioned in the introduction, Vejbæk (in press) has described seismic recordings from the Rønne Graben interpreted as indications of 4 to $5 \mathrm{~km}$ of presumed Palaeozoic deposits. Most of these are supposed to be of Late Silurian age. This conclusion is partly drawn from the knowledge of an $800-1200 \mathrm{~m}$ thick Late Silurian sequence found in Scania (Bergström et al. 1982). In support of a deep burial history are also the occurrence of pressure solution phenomena in the Hardeberga Sandstone Fm. on Bornholm, which suggests 
burial depths in excess of $1 \mathrm{~km}$ (Blatt et al., 1980, p. 360).

It is our opinion that the arguments listed above are in favour of a deep burial palaeothermal history, where geothermal heating reached at least $100^{\circ} \mathrm{C}$. This burial heating caused overmaturation of organic source-rock material, onset of metamorphic processes in clay minerals, colouring of conodonts and isotopic re-equilibration of the limestone/pore water system. An estimated minimal depth of burial can be calculated from the equation

$$
\mathrm{D}=\left(\mathrm{T}_{\mathrm{b}}-\mathrm{T}_{\mathrm{s}}\right) \times 1 / \mathrm{G}
$$

where $D$ is depth of burial in metres, $T_{b}$ is the burial temperature (min. estimate $100^{\circ} \mathrm{C}$ ). $T_{s}$ is the surface temperature in Lower Palaeozoic (max. estimate $30^{\circ} \mathrm{C}$ ) and $\mathrm{G}$ is the geothermal gradient in ${ }^{\circ} \mathrm{C} / 1000 \mathrm{~m}$. Depending on choice of $\mathrm{G}$, minimal calculated burial depths will vary from about $1500 \mathrm{~m}$ to $3000 \mathrm{~m}$. Actual depths were probably greater than the minimal estimate.

\section{Timing of the burial event}

It is the authors' opinion that the burial event leading to thermal diagenesis of the Lower Palaeozoic rocks of Bornholm is related to the later phases of the Caledonian orogeny. In favour of this interpretation are the following arguments:

1. Burial must have been post-Middle Silurian (total Cambro/Middle Silurian section on Bornholm is less than $500 \mathrm{~m}$ ).

2. Burial must have been pre-Lower Jurassic (Lower Jurassic organic matter is immature with respect to oil generation, Erik Thomsen, pers. comm. 1983).

3. In the well Slagelse 1 , shift from overmature to premature organic matter is found at the Silurian/Permian disconformity suggesting regional burial to be pre-Permian (Thomsen et al. 1983).

4. More than 800 of Late Silurian deposits occur in Scania, indicating accelerating subsidence in the region during this interval (Bergström et al. 1982). Further clarification of the timing problem must wait, however, until a more detailed subsidence model for the Bornholm area has been established.

\section{Formation and diagenetic evolution of the Lower Palaeozoic carbonates}

The investigated Lower Palaeozoic carbonate rocks from Bornholm have experienced a complex and until now only briefly investigated diagenetic evolution. The deep burial model enables us to propose a more detailed evolutionary framework than published hitherto. The following account which is illustrated graphically in fig. 7 summarizes the formational and diagenetical history of the Lower Palaeozoic carbonates as interpreted from the isotopic data.

\section{Precipitation of primary carbonates}

The primary carbonate units formed as biogenic and/or chemical precipitates from a marine water column with an carbon isotopic composition of dissolved bicarbonate slightly depleted in ${ }^{13} \mathrm{C}$ as compared to that of the present seas. The investi-
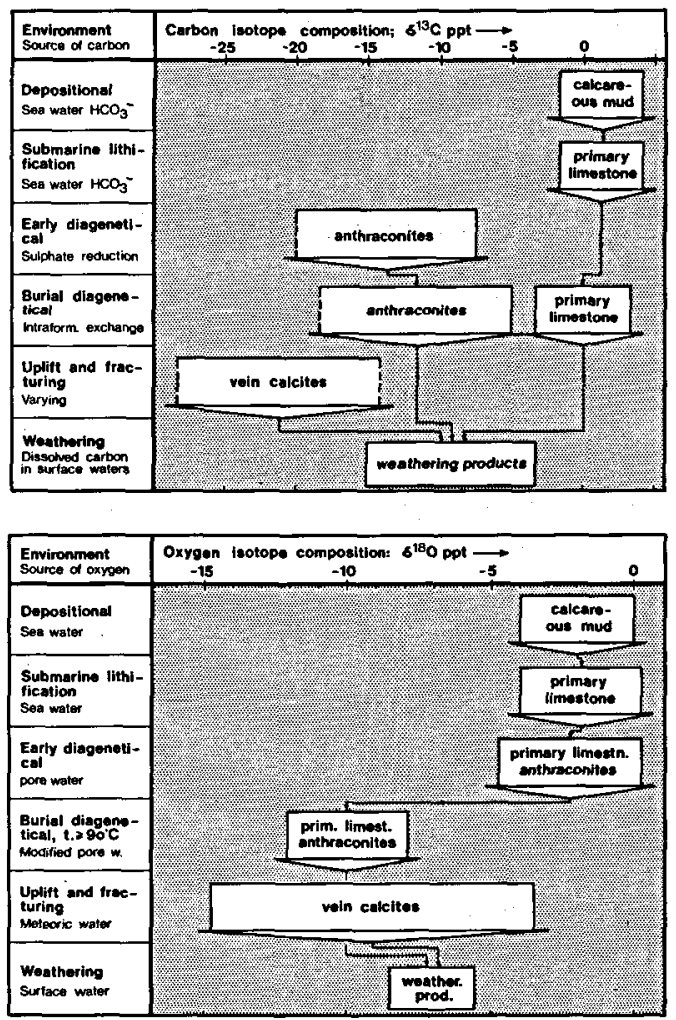

Figure 7. Depositional and diagenetical history of the investigated calcareous material as interpreted from the isotopic compositions. 
gated Silurian limestone beds probably also represent primary carbonate units. Partial lithification and formation of rigid framework took place syndepositionally.

\section{Formation of concretions}

During time intervals not favourable for the precipitation of primary carbonates, growth of early diagenetic concretions (anthraconites) took place in the uncompacted and water-filled, fine-grained detrital bottom sediments. Carbonate ions were produced from bacterial decomposition of organic material in the sediments. Sulphate reduction processes dominated over fermentation, and growth of concretions initiated within the uppermost few metres of the sediment column. Growth continued at deeper levels as indicated by internal structures of some of the anthraconitic concretions (cone-in-cone structures, shape of lamination). As rate of sedimentation was low during deposition of the black shale units $(<1 \mathrm{~cm} / 1000$ years), the time interval represented by growth of each individual concretion probably was in the order of million years.

The carbonate cementation of the Jerrestad Mudstone horizons probably took place early diagenetically, but may well have been initiated by a contribution of primary, depositional carbonate particles.

\section{Burial diagenesis}

During progressive burial of the sedimentary pile isotopic exchange processes took place between formation water and carbonates at increasing temperatures. The reactions ceased at burial temperatures of approx. $90^{\circ} \mathrm{C}$ owing to total expulsion of pore waters. This temperature corresponds to a burial depth of at least $1500 \mathrm{~m}-2000$ m. During the burial process limestone units were totally lithified. Further heating of the sediments did not produce any noticeable change of isotopic composition of the carbonates.

\section{Precipitation of vein clacites}

Following the main burial phase, gradual uplift and erosion of the sedimentary pile shifted the carbonate rocks into depth zones where precipitation of calcites in fracture zones could take place at lower temperatures. The source of water for these processes probably was meteoric, while carbonate carbon originated from different sources.

\section{Weathering}

After exposure to surface waters in Late to Post Glacial time, minor modifications took place that affected boundary zones between shale and limestone units and subaerically exposed surfaces.

\section{Summary and conclusion}

The present study reports carbon and oxygen isotope determinations of 71 samples of Lower Cambrian to Middle Silurian limestone, calcareous concretions, calcite cement and vein fillings. It is shown that the investigated material has preserved its original carbon isotope distribution except for minor modifications, but that the oxygen isotope composition has been totally modified by post-depositional processes. These modifications have been ascribed to burial diagenesis at elevated temperatures. If this hypothesis is accepted, the following conclusions can be drawn:

1. The original carbon isotope composition of a lithified limestone will be preserved even under severe diagenetic modifications at temperature/ depth conditions significantly different from those of the depositional environment. In the present case, the carbon isotope distribution reflects the difference between primary limestones formed in sea water and calcareous concretions (anthraconites) formed in the uppermost depth zones of the sea bottom sediments. The assumed early diagenetic origin of the anthraconitic concretions is thus confirmed.

2. The oxygen isotope composition of a fossil limestone may reflect conditions other than those of the primary depositional environment. Consequently proof must be provided for the preservation of original isotopic composition before any palaeo-environmental interpretations can be made from the actual oxygen isotope data. In the present case, the oxygen isotope composition reflects re-equilibration between water and minerals at temperatures in the range of $90^{\circ} \mathrm{C}$ or more. 
3. It is argued that the thermal influence observed from the oxygen isotope composition of the investigated calcareous material is due to geothermal impact from deep burial rather than from magmatic heating. Burial depths in excess of $2000 \mathrm{~m}$ is necessary to provide the suggested minimum temperatures. It is the authors' opinion that the major subsidence of the area took place in Late Silurian time. The thermal event can thus be related to the latest phases of the caledonian orogeny.

Acknowledgements. We want to express our gratitude to I. Nyegaard and B. Warming for preparatory work and to T. Cederberg for maintenance of the mass spectrometer. $\mathrm{R}$. G. Bromley and Erik Thomsen gave critical comments on the manuscript. This study was supported by the Danish Science Research Council (mass spectrometer facility, Grant no 119372) and the Danish Ministry for Energy Resources (Grant no. EFP-83, 2251-302). One of the authors (ATN) was supported by the Carlsberg Foundation.

\section{Dansk sammendrag}

De Kambro-Silure aflejringer på Bornholm omfatter ud over grov- og finklastiske bjergarter også kalksten. Disse kalksten er udviklede som typiske primære, marine kalksten eller som tidligt diagenetiske konkretioner (antrakonitter). Derudover findes calcitiske sprækkeudfyldninger. Iltisotopsammensætningen af de primære kalksten og af konkretionerne er homogeniseret set i relation til både lithologiske og stratigrafiske forskelle. De målte værdier er signifikant forskellige fra en sammensætning svarende til diagenetisk upåvirkede, marine kalksten af nedre Palæozoisk alder. Det er derfor nødvendigt at forklare den aktuelle iltisotopsammensætning som et resultat af "begravelses«diagenetiske processer ved temperaturer på $90^{\circ} \mathrm{C}$ eller derover. I modsatning hertil ses de målte kulstofisotop-sammensætninger at svare til originale, præ-diagenetiske vardier. De observerede forskelle afspejler oprindelige forskelle mellem calcium karbonat udfældet $\mathrm{i}$ ligevægt med marint bikarbonat og calcium karbonat dannet $i$ den sulfat-reducerende zone $i$ havbundssedimenterne. De calcitiske sprakkeudfyldninger har isotopsammensætninger, der både hvad kulstof og ilt angår er væsensforskellige fra det øvrige materiale. Afhængigt af valg af geotermisk gradient og primare aflejringsparametre kan den observerede termiske effekt forklares ved en generel indsynkning af den undersøgte sekvens til dybder på mindst $2 \mathrm{~km}$. Det foreslås, at denne indsynkning fandt sted i sen Silurisk til tidlig Devon tid i sammenhæng med den Kaledoniske hovedfase.

\section{References}

Abrahamsen, N. 1977: Palaeomagntism of 4 dolerite dikes around Listed, Bornholm. Bull. geol. Soc. Denmark 26, 195-215.

Allan, J. R. And Matthews, R. K. 1982: Isotope signatures associated with early meteoric diagenesis. Sedimentology 29 , 797-818.
Berg-Madsen, Vivianne 1981: The Middle Cambrian Kalby and Borregård Members of Bornholm, Denmark. Geol. För. Stockh. Förh. 103, 215-231.

Bergström, J., Holland, B., Larsson, K., Norling, E. and Sivhed, U. 1982: Guide to excursions in Scania. Sver. geol. Unders. Ser. ca. 54, 95 pp.

Berner, R. A. 1968: Chemical changes affecting dissolved calcium during the bacterial decomposition of fish and clams in sea water. Mar. Geol. 7, 253-274.

Bjerreskov, M. 1975: Llandoverian and Wenlockian graptolites from Bornholm. Fossils \& Strata, 8, $94 \mathrm{pp.}$

Bjørlykke, K. 1966: Sedimentary petrology of the Sparagmites of the Rena District, S. Norway. Norg. geol. Unders. 238, 5-53.

Blatt, H., Middleton, G. and Murray, R. 1980: Origin of sedimentary rocks. Prentice-Hall, New Jersey, 782 pp.

Brand, U. 1982: The oxygen and carbon isotope composition of Carboniferous fossil components: sea-water effects. Sedimentology 19, 139-148.

Buchardt, B. and Cederberg, T. (in prep): Carbon isotope composition of Lower Palaeozoic organic-rich shales from Bornholm.

Cocks, L. R. M. and Fortey, R. A. 1982: Faunal evidence for oceanic separation in the Palaeozoic of Britain. J. geol. Soc. $139,465-478$.

Coleman, M. L. \& Raiswell, R. 1981: Carbon, oxygen and sulphur isotope variations in concretions from the Upper Lias of N. E. England. Geochim. cosmochim. Acta 45, 329 340 .

Dansgaard, W. 1964: Stable isotopes in precipitation. Tellus 16, 436-468.

Degens, E. T. 1969: Biogeochemistry of stable carbon isotopes In: Eglinton, G. \& Murphy, M. T. J. (eds): Organic Geochemistry, 304-329. Springer Verlag, Berlin.

Deines, P. 1980: The isotopic composition of reduced organic carbon. In: Fritz, P. \& Fontes, J. C. (eds): Handbook of environmental isotope geochemistry, vol. 1, The terrestrial environment, A. 329-406. Elsevier, Amsterdam.

Epstein, S., Buchsbaum, R. Lowenstam, H. A. and Urey, H. C. 1951: Carbonate-water isotopic temperature scale. Bull. geol. Soc. Am. 62, 417-425.

Eslinger, E. V. and Savin, S. M. 1973: Oxygen isotope geothermometry of the burial metamorphic rocks of the Precambian Belt Supergroup, Glacier National Park, Montana. Bull. geol. Soc. Am. 84, 2549-2560.

Forester, R. W. and Taylor, H. P. 1977: ${ }^{18} \mathrm{O} /{ }^{16} \mathrm{O}, \mathrm{D} / \mathrm{H}$ and ${ }^{13} \mathrm{C}$ ${ }^{12} \mathrm{C}$ studies of the Tertiary igneous complex of Skye, Scotland. Am. J. Sci. 277, 136-177.

Gee, D. G. 1972: The regional geological context of the Tåsjö uranium project, Caledonian, Central Sweden. Sver. geol. Unders. Ser. C. 671, 1-36.

Gross, M. G. 1964: Variations in the $\mathrm{O}^{18} / \mathrm{O}^{16}$ and $C^{13} / C^{12}$ ratios of diagenetically altered limestones in the Bermuda Islands. J. Geol. 72, 170-194.

Grönwall, K. A. 1899: Bemærkninger om de sedimentære Dannelser paa Bornholm og deres tektoniske Forhold. Danm. geol. Unders. II $r k, 10,1-48$.

Grönwall, K. A. 1902: Bornholms Paradoxideslag og deres Fauna. (Summary in English). Danm. geol. Unders. II $r k$, $13,230 \mathrm{pp}$

Hansen, K. 1945: The Middle and Upper Cambrian sedimentary rocks of Bornholm. Danm. geol. Unders. II rk, 72, 81 pp.

Irwin, H. and Curtis, C. 1977: Isotopic evidence for source of diagenetic carbonates formed during burial of organic-rich sediments. Nature 269, 209-213.

Keith, M. L. and Weber, J. N. 1964: C and O isotopic composition of selected limestones and fossils. Geochim. cosmochim. Acta, 28, 1787-1816. 
Klingspor, I. 1976: Radiometric age determinations of basalts, dolerites and related syenite in Skåne, southern Sweden. Geol. Fören. Stockh. Förh. 98, 195-216.

Lindström, M. 1984: The ordovician climate based on the study of carbonate rocks. In: Bruton, D. L. (ed.): Aspects of the Ordovician System, 81-88. Palaeontological Contributions from the University of Oslo, No. 295. Universitetsforlaget.

Magaritz, M. 1974: Lithification of chalky limestone: a case study in Senonian rocks from Israel. J. sedim. Petrol. 44, 947-954.

McCrea, J. M. 1950: The isotopic chemistry of carbonates and a paleotemperature scale. J. chem. Phys. 18, 849-857.

Mook, W. G. 1971: Paleotemperatures and chlorinities from stable carbon and oxygen isotopes in shell carbonate. $\mathrm{Pal}$ aeogeogr. Palaeoclimatol., Palaeoecol. 9, 245-263.

Nielsen, A. T. (in prep): The stratigraphy and palaeoecology of the Lower Ordovician Komstad Limestone, southern Scandinavia.

Oftedahl, C. 1945: Om tilliteme i det central-norske sparagmitområde. Norsk geol. Tidsskr. 25, 285-293.

O'Neill, J. A., Clayton, R. N. and Mayeda, T. K. 1969: Oxygen isotope fractionation in divalent metal carbonates. $J$. chem. Phys. 51, 5547-5558.

Perry, E. C. jr. 1967: The oxygen isotope chemistry of ancient cherts. Earth planet. Sci. Lett. 3, 62-66.

Poulsen, C. 1923: Bornholms Olenuslag og deres fauna. Danm. geol. Unders. II $r k, 40,83 \mathrm{pp}$.

Poulsen, C. 1936: Übersicht über das Ordovizium von Bornholm. Meddr. dansk geol. Foren. 9, 43-66.

Poulsen, V. 1965: An early Ordovician trilobit fauna from Bornholm. Meddr. dansk geol. Foren. 16, 49-115.

Poulsen, V. 1966: Cambro-Silurian stratigraphy of Bornholm. Meddr. dansk geol. Foren. 16, 117-137.

Poulsen, V. 1978a: The Precambrian-Cambrian boundary in parts of Scandinavia. Geol. Mag. 115, 131-136.

Poulsen, V. 1978b: Dalmanitina beds (late Ordovician) on Bornholm. Danm. geol. Unders. Arbog 1976, 53-88.

Raiswell, R. 1972: The growth of Cambrian and Liassic concretions. Sedimentology, 17, 147-171.
Raiswell, R. 1976: The microbiological formation of carbonate concretions in the Upper Lias of NE England. Chem. Geology, 18, 227-244.

Schidlowski, M., Eichmann, R. and Junge, C. E. 1975: Precambrian sedimentary carbonates: carbon and oxygen isotope geochemistry and implications for the terrestrial oxygen budget. Precamb. Res. 2, 1-69.

Shackleton, N. J. and Kennett, J. P. 1975: Paleotemperature history of the Cenozoic, and the initiation of Antarctic glaciation: oxygen and carbon isotope analyses in DSDP sites 277, 279 and 281. Initial reports of the Deep Sea Drilling Project 29, 743-755.

Spjeldnæs, N. 1981: Lower Palaeozoic palaeoclimatology. In: Holland, C. H. (ed.): Lower Palaeozoic of the Middle East, Eastern and Southern Africa and Antarctica. 199-256. Wiley, New York.

Surlyk, F. 1980: Geology of the European countries. Denmark. C. N. F. G. 26th Int. Geol. Congr., 5-50. Dunod, Paris.

Taylor, H. P. 1977: Water/rock interactions and the origin of $\mathrm{H}_{2} \mathrm{O}$ in granitic batholiths. J. geol. Soc. 133, 509-558.

Thomsen, E., Lindgreen, H. and Wrang, P. 1983: Investigation on the source rock potential of Denmark. Geologie Mijnb. $62,221-239$.

Veizer, J. and Hoefs, J. 1976: The nature of ${ }^{18} \mathrm{O} /{ }^{16} \mathrm{O}$ and ${ }^{13} \mathrm{C} /{ }^{12} \mathrm{C}$ secular trends in sedimentary carbonate rocks. Geochim. cosmochim. Acta 40, 1387-1395.

Veizer, J., Holser, W. T. and Wilgus, C. K. 1980: Correlation of ${ }^{13} \mathrm{C}^{12} \mathrm{C}$ and ${ }^{34} \mathrm{~S} /{ }^{32} \mathrm{~S}$ secular variations. Geochim. cosmochim. Acta 44, 579-588.

Vejbæk, O. (in press): Seismic stratigraphy and tectonics of sedimentary basis around Bornholm, southern Baltic. Danm. geol. Unders.

Walls, R. A., Mountjoy, E. W. and Fritz, P. 1979: Isotopic composition and diagenetic history of carbonate cements in the Devonian Golden Spike reef, Alberta, Canada. Bull. geol. Soc. Am. 90, 963-982.

Webby, B. D. 1984: Ordovician reefs and climate, a review. In: Bruton, D. L. (ed): Aspects of the Ordovician system. Palaeontological Contributions of the University of Oslo, 89100. Universitetsforlaget, Oslo. 